\title{
Comportement des remblais en enrochement
}

\section{E. ALONSO}

Universidad Politécnica

de Catalunya

Barcelone (Espagne)

\section{OLDECOP}

Universidad nacional de San Juán

San Juán (Argentine)

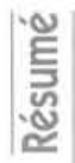

Les résultats des essais en laboratoire et l'expérience in situ démontrent que les remblais en enrochement se déforment au cours du temps selon un processus qui est lié à l'action de l'eau. Les essais réalisés dans des cellules œdométriques démontrent que l'humidité relative de l'air dans les vides des enrochements est la variable qui contrôle I'action de I'eau. Les phénomènes de propagation subcritique de fissures offrent un cadre de référence conceptuel adéquat pour développer des modèles constitutifs macroscopiques. Un modèle basé sur la théorie de la plasticité avec écrouissage est développé afin de reproduire le comportement des essais de compression unidimensionnelle réalisés. Le modèle constitutif est couplé à des phénomènes d'écoulement pour développer un outil réaliste qui puisse résoudre des problèmes aux limites. Cet article décrit un exemple dans lequel l'histoire complète d'un essai cedométrique a été simulée. Il semble que le modèle développé offre une meilleure compréhension du comportement des structures en enrochement.

Mots-clés : relations constitutives, essais en laboratoire, gravier, enrochement, succion, compressibilité, flux.

\section{Behaviour of rockfill embankments}

Both, laboratory test results and field experience show that rockfill embarikments deform along time in a process that is linked to water action. Tests performed in oedometer cells have shown that the relative humidity of the air within the rockfill voids is the variable that controls water action. Subcritical crack propagation phenomena provide an adequate conceptual reference framework to develop macroscopic constitutive models. A model has been developed within the theory of hardening plasticity. It explains the behaviour of the onedimensional tests performed. The constitutive model was coupled with flow phenomena in order to develop a realistic tool to solve boundary value problems. The paper reports an example in which the complete history of an oedometer test has been simulated. It is thought that the model developed offers a better understanding of the behaviour of rockfill structures.

Keywords ; constitutive relations, laboratory tests, gravels, rockfill, suction, compressibility, flow. 


\section{Introduction}

L'emploi de remblais en enrochement dans la construction est probablement aussi ancien que notre civilisation. Un exemple d'une étonnante modernité est le barrage de Sadd-el-Kaffara (Fig. 1) construit en Egypte en 2600 av. J.-C. (Schnitter, 1994). Le barrage, de $14 \mathrm{~m}$ de hauteur, avait un noyau imperméable protégé par cleux épaulements en enrochement rattachés au parement par un ouvrage de maçonnerie. Mais, c'est seulement au début du $\mathrm{XX}^{e}$ siècle que se généralise la conception de barrages comportant soit des recharges, soit l'ensemble du profil résistant en enrochement. Dans ces grandes structures, on observait fréquemment des tassements différés ou rapides, ces derniers étant associés à l'immersion des zones d'enrochements. Il fut vérifié que le compactage en couches des remblais, comme dans les terre-pleins, réduisait considérablement les tassements. Ainsi les données de Sherard et Cooke (1987) montrent que les tassements, après construction dans des enrochements compactés, représentent $0,13 \%$ de la hauteur totale de l'ouvrage, tandis qu'elles atteignent $0,75 \%$ de la hauteur et voire plus dans le cas d'enrochements non compactés.
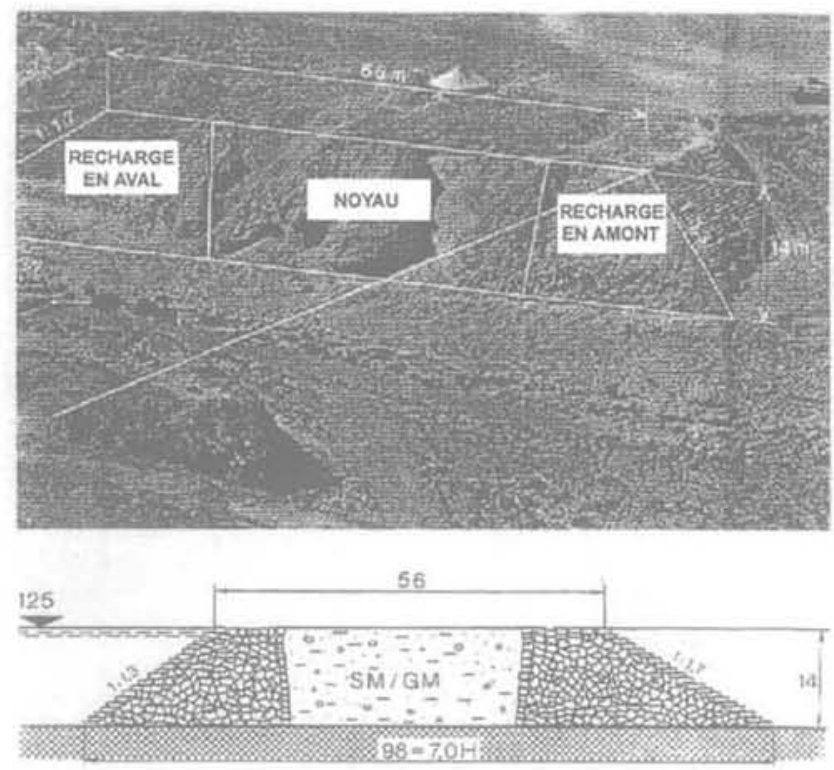

FIG. Sadd-el-Kaffara. Barrage en enrochement construit en Égypte en I'an 2600 av. J.-C. (Schnitter, 1994)

Sadd-el-Kafara. Rockfill dam built in Egypt 2600 a.C (Schnitter, 1994).

Les tassements progressent indéfiniment avec le temps. Les mesures de tassements recueillies par Sowers et al. (1965) en crête de barrages en enrochement à partir de la fin de la construction mettent en évidence la tendance des tassements différés à croître linéairement (et parfois à une vitesse supérieure) avec le logarithme du temps.

La présence d'eau a une importance capitale dans la génération des tassements. On connaît bien les phénomènes d'effondrement des enrochements en amont des barrages lors de la première mise en eau. Le terme " effondrement $»$ signifie dans ce contexte l'augmentation de la déformation à contrainte de confinement constante. Les phénomènes d'effondrement de maté- riau granulaire grossier ont été observés dans des barrages et des remblais comme en laboratoire (Sowers et al., 1965; Marsal, 1973; Nobari et Duncan, 1972). Les figures $2 \mathrm{a}$ et $2 \mathrm{~b}$ reproduisent les résultats des essais œdométriques publiés par Nobari et Duncan (1972) à propos d'une argilite triturée et compactée provenant du barrage Pyramid. La saturation des échantillons initialement secs, à contrainte constante (Fig. 2a) ou à porosité constante (Fig, 2b) conduit le volume ou l'état de contrainte de l'échantillon à la courbe de compression saturée. Ce résultat est formellement identique à celui observé pendant la saturation des sols non saturés (Alonso et al., 1987).
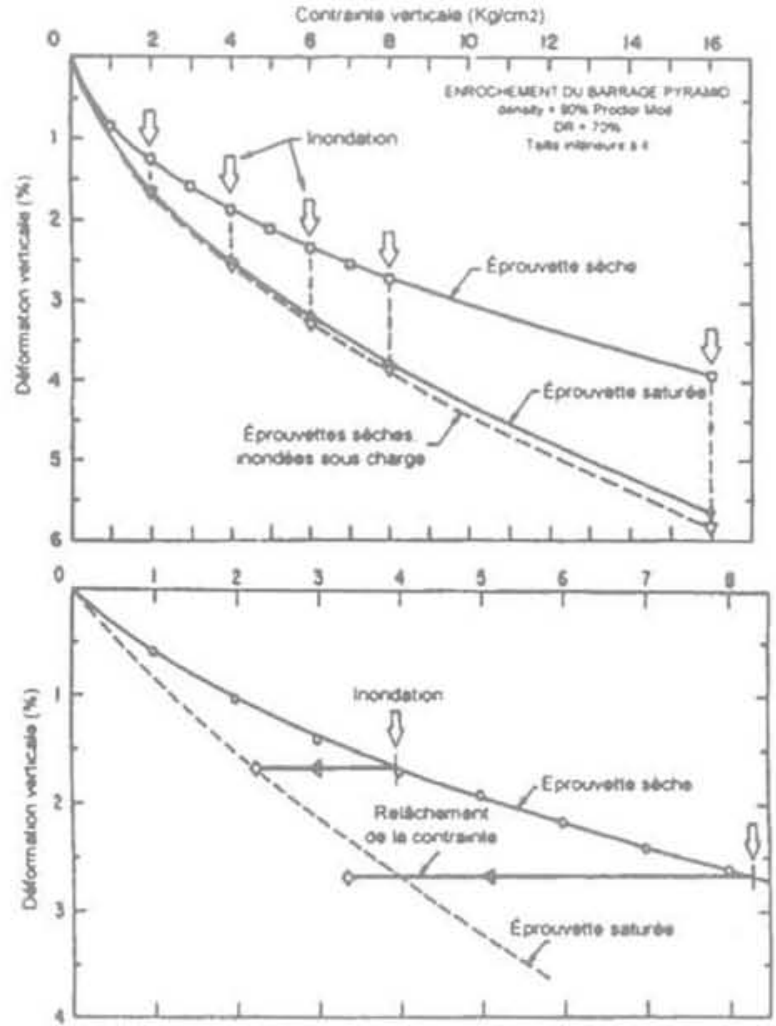

FG. 2 Essais de Nobari et Duncan (1972). Inondation à contrainte constante (a) ou à volume constant (b).

Nobari and Duncan (1972) tests. Flooding at constant stress (a) or constant volume (b).

Toutefois, les mécanismes de déformation des matériaux granulaires grossiers et des sols fins (sable fin et tailles inférieures) doivent être radicalement différents. Ceci est illustré sur la figure 3 où l'on a dessiné la variation de la force d'attraction capillaire entre deux particules sphériques en contact avec un ménisque d'eau de courbure (et donc de succion) variable. La force d'attraction capillaire, $F_{w}$, est calculée en employant la théorie du ménisque toroïdal présentée par Gili (1988). Dans les quatre cas représentés (enrochement : diamètre $\mathrm{D}=50 \mathrm{~cm}$; gravier : $\mathrm{D}=10 \mathrm{~mm}$; sable moyen : $\mathrm{D}=0,5 \mathrm{~mm}$ et limon moyen : $\mathrm{D}=0,01 \mathrm{~mm}$ ), on a employé le poids du grain comme référence pour normaliser la valeur de $\mathrm{F}_{\mathrm{w}}$. On observe que, pour les tailles supérieures au gravillon, le poids de chaque grain surpasse nettement la force capillaire maximale aux contacts isolés et donc les forces internes d'origine capillaire tendront à être négligeables dans les matériaux grossiers. 


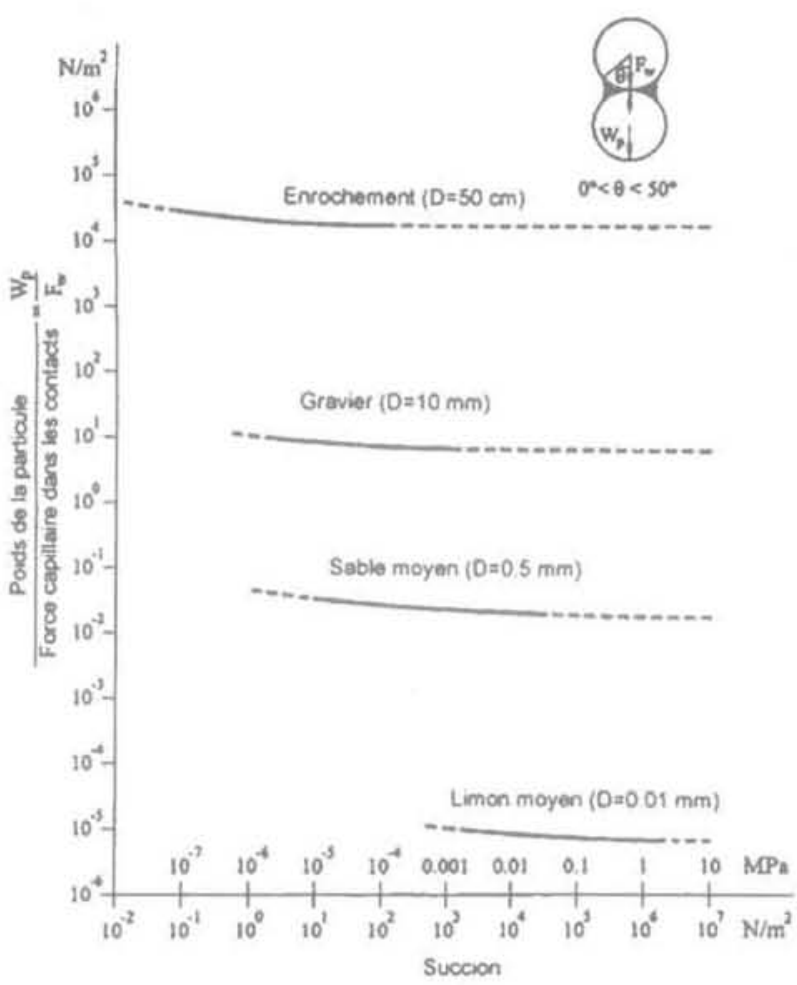

FiG. 3 Force d'attraction capillaire entre sphères en contact.

Capillary attraction force between spheres in strict contact.

Sur la figure 4, on a représenté la valeur absolue des forces capillaires $F_{\text {w }}$ en fonction de la contrainte normale moyenne le long d'un plan de référence qui passe par les contacts entre particules. Pour cela, on a fait l'hypothèse simple d'une configuration cubique de sphères. La réduction rapide du nombre de contacts par unité de surface fait que les contraintes équivalentes à la succion capillaire dans les matériaux grossiers soient très petites. Ceci est illustré sur la figure 4 dans le cas de quatre matériaux granulaires choisis à cette fin. En conclusion, les contraintes d'origine capillaire ne sont pas suffisantes pour expliquer l'effet de l'eau dans les matériaux granulaires grossiers.

Des essais de compression, réalisés par des différents auteurs (Sowers et al., 1965, Marsal, 1973, Clements, 1981) sur des pointes isolées de roche en contact avec des surfaces du même ou d'autres matériaux, suggèrent que l'écrasement des contacts est la principale cause qui explique la déformation de l'enrochement ainsi que l'effet de l'eau. Sur la figure 5, on reproduit l'essai de compression présenté par Clements (1981) sur une cale de roche d'angle au sommet $169,1^{\circ}$. La saturation de la pointe, effectuée deux heures après l'application de la charge de compression, produit une accélération brusque de la déformation. Sowers et al. (1965) ont observé le même comportement dans des essais œdométriques sur échantillons granulaires de Grauwacke et de grès (Fig. 5).

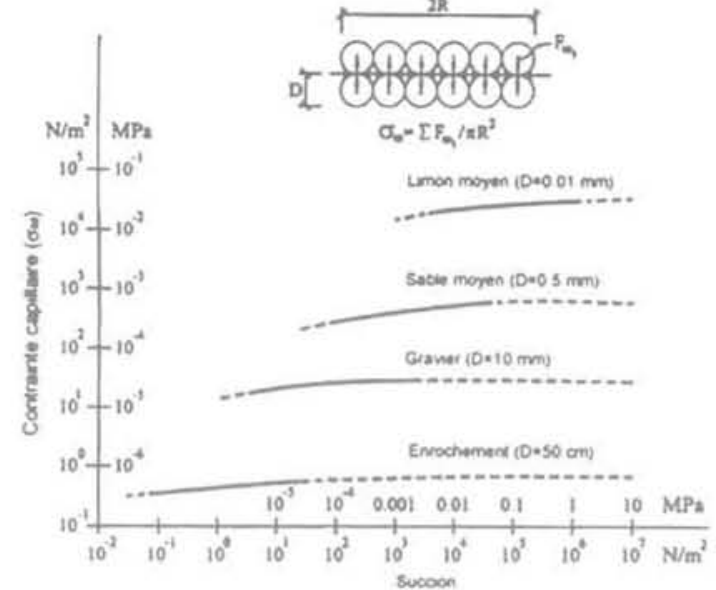

FG.4 Contrainte d'attraction capillaire dans une configuration de sphères de porosité maximale.

Capillary attraction stress in a configuration of spheres having maximum porosity.
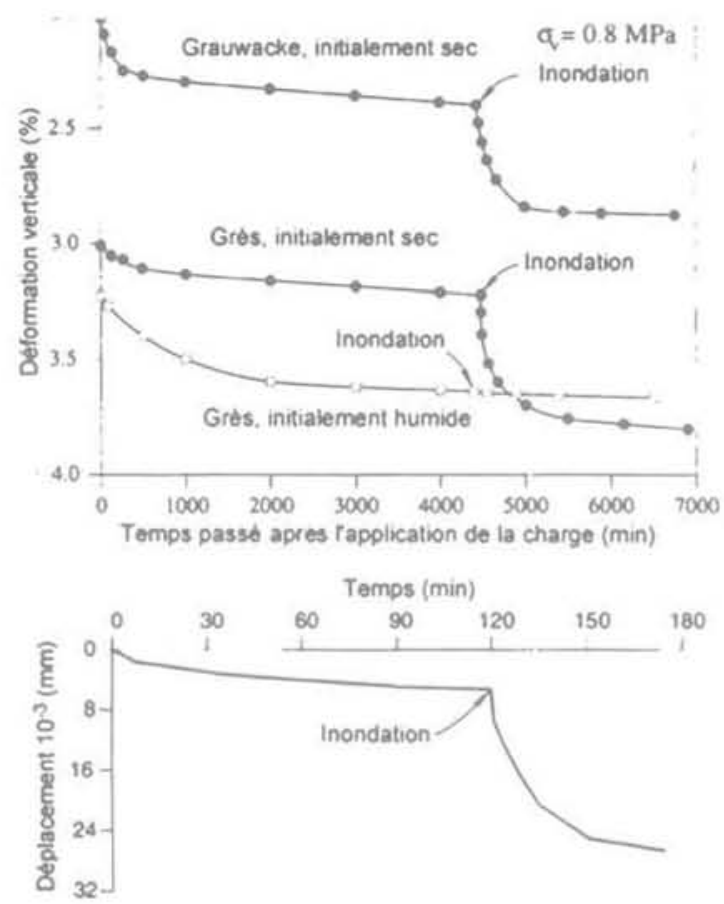

Fla 5 (a) Effet de l'immersion dans les essais de compression monodimensionnels de roche broyée sous contrainte verticale constante égale à $0,8 \mathrm{MPa}$ (Sowers et al., 1965). Diamètre de codomètre $=190 \mathrm{~mm}$, hauteur de l'éprouvette $=100 \mathrm{~mm}$, taille maximale des particules $=38 \mathrm{~mm}$. (b) Essai d'inondation d'une pointe de roche (Clements, 1981).

(a) Effect of flooding in one-dimensional compression tests of crushed rock under a constant vertical stress ( $0.8 \mathrm{MPa}$ ) (Sawers et al., 1965). Oedometer diameter $=190 \mathrm{~mm}$; specimen thickness $=100 \mathrm{~mm}$ : maximum size of particles $=38 \mathrm{~mm}$. (b) Flooding test on a rock point (Clements, 1981). 
Terzaghi (1960) a été l'un des premiers à suggérer que la déformation des enrochements est causée par l'écrasement de particules à proximité de contacts très surchargés et par la postérieure remise en ordre de la structure granulaire vers une configuration plus stable. Les essais mentionnés ci-dessus montrent de plus que la présence d'eau accélère l'écrasement des contacts et donc la déformation globale du matériau granulaire.

La saturation des enrochements n'est pas la seule cause des effondrements observés dans les recharges ou barrages en enrochement. L'action des pluies, incapables de saturer ces matériaux à forte perméabilité, a des effets similaires. Ainsi, Naylor et al. (1997) ont montré un rapport direct entre les tassements du barrage de Beliche et l'intensité des précipitations. Bauman (1960) a décrit comment le barrage de Logswell connut de graves tassements d'effondrement lors d'une pluie de $360 \mathrm{~mm}$. Dans ce barrage à masque en béton, le corps du remblai a été construit avec un gneiss granitique non préalablement compacté ou mouillé. Suite à la pluie, les tassements verticaux ont atteint $4 \%$ de la hauteur du barrage, ce qui a causé l'écrasement du masque en béton.

La figure 6 illustre le comportement du barrage de l'Infernillo, d'une hauteur de $150 \mathrm{~m}$, à la fin de sa construction. C'est un barrage à noyau central en argile et recharges en enrochement de diorite et conglomérats, déversés secs et sans compactage. En suivant le comportement de la borne $\mathrm{M}-10$, on peut observer l'effondrement de la recharge amont pendant la première mise en eau en 1964. Il est de plus significatif qu'après 18 mois de vitesse décroissante de déformation (de janvier 1965 à juillet 1966) on observe une soudaine augmentation des tassements et des déplacements horizontaux des bornes M-10 et M-23. Cette accélération des mouvements est visible jusqu'à la fin de 1968 et correspond aux périodes de pluie intense qui ont commencé en 1966. On peut aussi vérifier que le tassement additionnel mesuré en crête du barrage à partir du premier remplissage, en apparence dû aux pluies et à la prévisible humidification progressive de la recharge amont, est du même ordre de magnitude que le tassement initial d'effondrement induit par la saturation complète de la recharge en amont. La vitesse de ce deuxième tassement différé est seulement plus lente.

Au contraire des épaulements amont des barrages, les remblais employés sous les voies de communication se verront rarement inondés. Mais ils seront soumis à l'action des pluies et on peut prévoir qu'ils subjront des phénomènes similaires à ceux observés à 1'Infernillo. Soriano et Sánchez (1999) ont décrit le comportement après construction des remblais de la ligne de train à haute vitesse Madrid-Séville, majoritairement construits en enrochements d'ardoise et de schiste. L'un des cas décrits, qui correspond à un remblai d'une hauteur de $40 \mathrm{~m}$, est représenté à la figure 7 . On observe dans ce cas un rapport clair entre l'intensité de la pluie et la vitesse du tassement pendant les années 1996-1997. Il est de plus intéressant de noter que les pluies additionnelles de la fin de 1997, d'une intensité similaire à celle de la fin de 1996, ne produisent plus de mouvements significatifs. On pourrait conclure que les précipitations antérieures ont préconsolidé le remblai.

L'interprétation de mesures in situ ainsi que les programmes d'essai dans des équipements de grand diamètre ont permis une connaissance raisonnable de la nature de la déformation dans les matériaux granu-
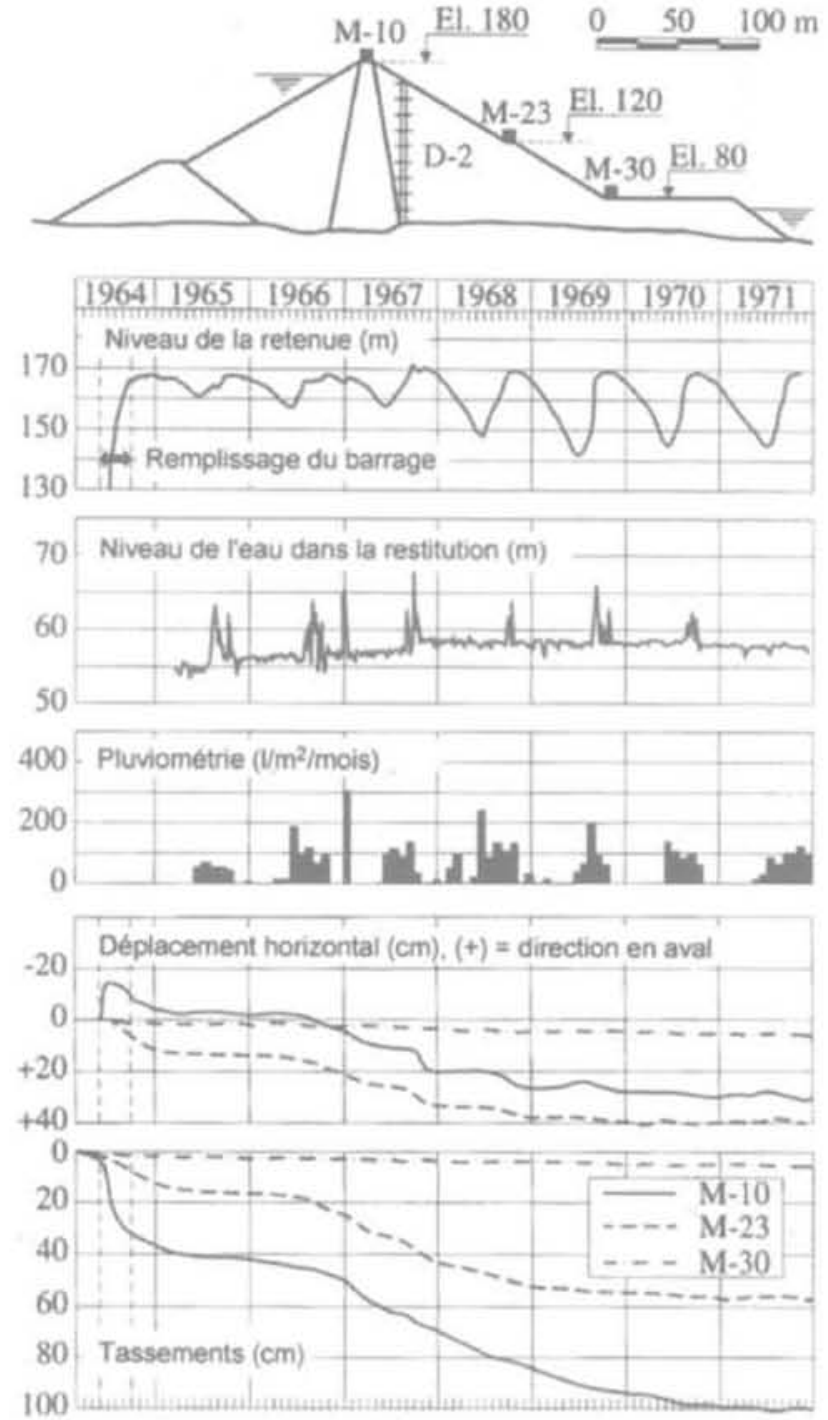

FIG.6 Lectures de tassements et déplacements horizontaux de bornes topographiques sur le barrage d'El Infernillo (Marsal et al., 1976).

Settlement and horizontal displacement records at topographic marks in El Infernillo dam (Marsal et al,, 1976).

laires grossiers. Les principaux programmes expérimentaux publiés correspondent à des essais œdométriques et triaxiaux. D'autres essais ont aussi été réalisés dans des cellules à déformation plane et de cisaillement en torsion. La recherche expérimentale en laboratoire la plus intense a eu lieu au cours des années 1960, 1970 et 1980. Au cours des années suivantes, l'intérêt a diminué. Il est intéressant de noter que la plus grande taille de particules testée en laboratoire est de $20 \mathrm{~cm}$ et correspond aux essais réalisés par Marsal (1973).

Dans tous les essais, une rupture significative des particules fut observée. Elle dépend du niveau de contraintes atteint, de la forme des particules, de la granulométrie (les particules anguleuses et les matériaux de granulométrie uniforme sont ceux qui ont la plus grande propension à la rupture), de la résistance de la roche et de la présence d'eau. L'effet d'échelle fut aussi examiné, plus concrètement par Marachi et al. (1969). Il se traduit par une rupture plus intense des particules 


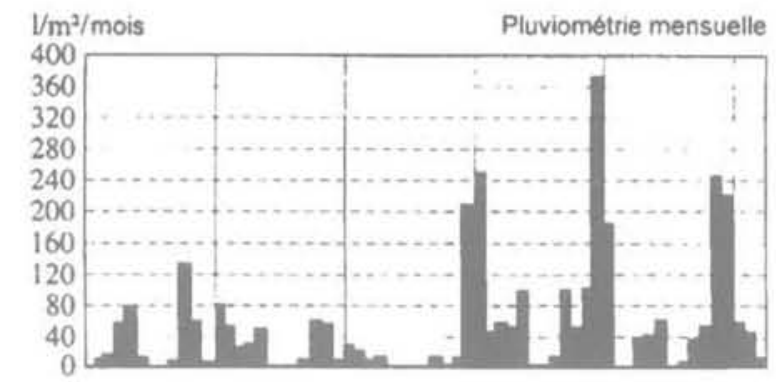

$\mathrm{mm} / \mathrm{mois}$

Vitesse de tassement

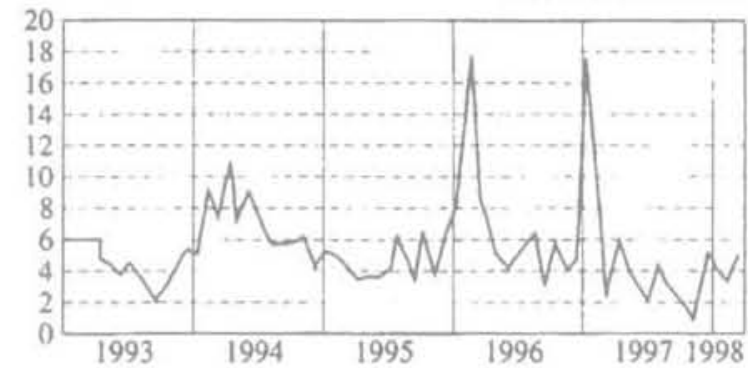

FIG 7 Lectures de vitesse de tassements d'un remblai de $40 \mathrm{~m}$ d'hauteur sous la voie du train de haute vitesse (TGV) Madrid-Séville et pluviométrie mensuelle sur le site (données de Soriano et Sánchez, 1999).

Settlement rate records for a $40 \mathrm{~m}$ thick rockfill embankment belonging to the High Speed Railway link Madrid-Sevilla and rainfall monthly records at the site (Soriano and Sánchez, 1999).

dans les matériaux les plus grossiers. L'effet de l'eau a été étudié de façon détaillée par Nobari et Duncan (1972) à partir d'essais œdométriques et triaxiaux sur argilite triturée. Ils ont observé que la teneur en eau initiale est le facteur le plus important pour déterminer l'intensité de l'effondrement en cas d'inondation. Plus la teneur en eau est haute, moins grand est l'effondrement en cas d'inondation. Cela confirme l'importance de la pratique habituelle d'arroser l'enrochement pendant la phase de compactage. Ils ont en outre montré que la rupture des particules se produit suite à l'application d'incréments de charge ou après un processus d'effondrement à contrainte de confinement constante.

L'ensemble de ces résultats expérimentaux n'a toutefois pas conduit à l'élaboration de modèles complets de comportement, ni à l'incorporation d'une façon claire de l'action de l'eau. Cela n'a pas empêché la réalisation d'analyses numériques du comportement des barrages et des remblais en employant la technique des éléments finis et des modèles contrainte-déformation relativement simples : modèle hyperbolique (Nobari et Duncan, 1972; Veiga Pinto, 1933; Soriano et Sánchez, 1996) ou modèles K-G (Naylor et al., 1986, 1997 ; Alonso et al., 1988). L'effet de l'eau y est pris en compte moyennant des artifices numériques.

L'analyse de Nobari et Duncan (1972) est probablement la première à prendre en compte cet effet. Leur méthode considère deux séries de paramètres rhéologiques, chacun correspondant au modèle hyperbolique. Le premier décrit le matériau à sa teneur d'eau initiale et le deuxième à sa teneur en eau finale (généralement à saturation), L'effet de la saturation est alors simulé en introduisant des forces nodales égales à la relaxation des contraintes, ce qui implique de « sauter ») de la série de paramètres à l'état sec à celle à l'état humide. Cette procédure a été employée par d'autres auteurs, cités auparavant. Naylor et al. (1986) généralisèrent la procédure à n'ímporte quel modèle rhéologique, y compris les modèles élastoplastiques. Alonso et al. (1993) décrivirent les déformations volumétriques des enrochements par deux surfaces d'état, qui utilisent une formulation propre aux sols non saturés pour introduire les déformations d'effondrement comme déformations initiales. Justo et Durand (1999) ont simulé les déformations différées des enrochements en employant un modèle viscoélastique monodimensionnel.

Dans cet article, on décrit les développements récents permettant de comprendre plus précisément l'action de l'eau sur les enrochements. Ils conduisent à la formulation de modèles constitutifs plus généraux qui, semble-t-il, permettent de reproduire d'une façon satisfaisante les résultats expérimentaux. Ainsi, une voie est ouverte pour une modélisation numérique plus sophistiquée des structures et des remblais en enrochement, qui intègre dans un cadre cohérent les actions environnementales et proprement mécaniques.

\section{2}

\section{Un modèle conceptuel de déformation pour les enrochements}

Divers phénomènes ont été proposés pour représenter la réduction de la résistance des roches due à l'action de l'eau: la perte de cohésion causée par la réduction de l'énergie superficielle des minéraux (Vutukuri et Lama, 1978); la réduction de la succion (Vutukuri et Lama, 1978) ; l'expansion des minéraux argileux (De Alba et Sesana, 1978; Delgado et al., 1982). Toutefois, ce sont les phénomènes de propagation sub-critique de fissures qui semblent le mieux s'adapter aux observations expérimentales.

Dans la théorie classique de la mécanique de la rupture, la propagation rapide et catastrophique d'une fissure se produit lorsque le facteur dit d'intensité des contraintes $(\mathrm{K})$ équivaut à une valeur critique (Kc), qui est une propriété du matériau connu sous le nom de ténacité. La valeur de K dépend de la géométrie de la fissure, du mode de chargement (traction, cisaillement normal ou parallèle à la fissure) et de l'intensité de la contrainte appliquée. Pour un mode de chargement (décrit par l'indice L) la valeur de K se calcule comme :

$$
K_{L}=\beta \sigma \sqrt{\pi a}
$$

où a est la longueur de la fissure (voir Fig. 8), $\sigma$, la contrainte de traction dans le cas représenté sur la figure 8 , et $\beta$, une constante qui dépend de la géométrie. Le mode $\mathrm{I}(\mathrm{L}=\mathrm{I})$ correspond à la traction pure ; $\mathrm{L}=\mathrm{II}$, au cisaillement normal à la fissure, et $\mathrm{L}=\mathrm{III}$, au cisaillement parallèle à la fissure. Le cas le plus habituel correspond à la propagation de la rupture en mode I. 


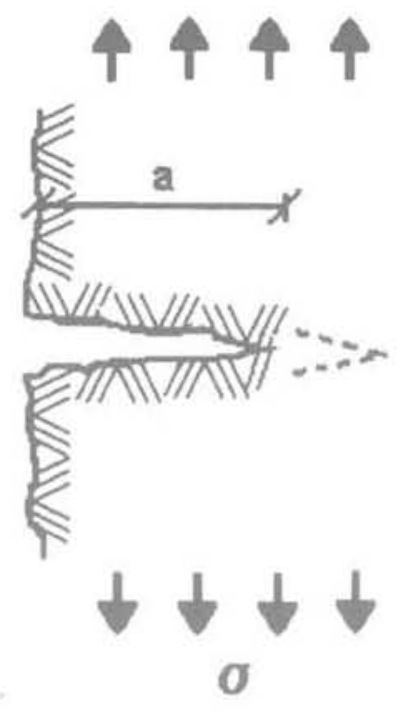

Géométrie d'une fissure.

Fissure geometry.
Il a été toutefois observé que la rupture peut se propager à une certaine vitesse $\mathrm{V}$, même quand $\mathrm{K}<\mathrm{Kc}$ (on omet l'indice L pour simplifier). Ce phénomène est connu comme propagation sub-critique de la rupture. La vitesse $V$ dépend de la valeur $K$, mais aussi de l'action d'agents (c corrosifs » externes comme l'eau. Les études de corrosion sous contraintes (Michalske et Freiman, 1982; Atkinson et Meredith, 1987) aident à interpréter le mécanisme de l'action de l'eau sur la propagation des ruptures. Dans le cas des minéraux de quartz, l'eau affaiblit les liaisons tendues silice-oxygène (Freiman, 1984) :

$$
\mathrm{H}_{2} \mathrm{O}+[\mathrm{Si}-\mathrm{O}-\mathrm{Si}] \rightarrow \chi \mp \rightarrow 2[\mathrm{Si}-\mathrm{OH}]
$$

La réaction conduit alors au complexe activé $\chi \neq$, dont les liaisons, plus faibles que celles du matériau d'origine, se brisent sous les contraintes appliquées. La vitesse de propagation de la fracture $V$ est contrôlée par la vitesse de la réaction (2). Des données expérimentales, similaires à celles représentées sur la figure 9 , indiquent que $\mathrm{V}$ dépend du facteur d'intensité des contraintes $\mathrm{K}$ et de l'humidité relative $(\mathrm{RH})$ qui est une mesure de l'énergie de l'eau. La formulation thermodynamique de la théorie de la vitesse de réaction appliquée à la corrosion sous contraintes en présence d'eau agissant comme agent corrosif (Widerhorn et al., 1980, 1982. Freiman, 1984) donne la structure suivante de dépendance entre $\mathrm{V}, \mathrm{K}$ et la $\mathrm{RH}$ :

$$
V=V_{o}(R H) \exp [-(E \mp-b K) / R T]
$$

Le terme $\left(\mathrm{E}_{+}^{+}-\mathrm{bK}\right)$ est interprété comme étant l'énergie d'activation (ou écran d'énergie) de la réaction de corrosion. Eł englobe les termes d'énergie qui ne dépendent pas de la contrainte. Le terme bK est inter- prété comme étant le travail des contraintes entre l'état initial et l'état activé. R est la constante des gaz parfaits et $\mathrm{T}$ la température absolue. Les paramètres $\mathrm{V}_{\gamma}$ EҒ et b sont des constantes pour un environnement déterminé, caractérisé par T et RH. L'équation (3) est cohérente avec les données expérimentales de la figure 9 (qui correspond à la propagation d'écrasement du verre) et aussi avec des données disponibles sur des roches (Atkinson et Meredith, 1987). Dans tous ces cas, on a observé que $\mathrm{V}$ augmente proportionnellement à $\mathrm{RH}$ (pour RH compris entre 20 et $80 \%$ ). Si la propagation de la fissure se produit dans un milieu liquide (par exemple une solution), on a observé expérimentalement (Freiman, 1984) que la vitesse de propagation est proportionnelle à I'humidité relative du gaz en équilibre thermodynamique avec le liquide. Dans ce cas, les potentiels chimiques de la solution et du gaz sont égaux. La figure 9 indique que les données de V correspondant à $\mathrm{HR}=100 \%$ sont très proches de ceux obtenus pour des éprouvettes plongées dans de l'eau.

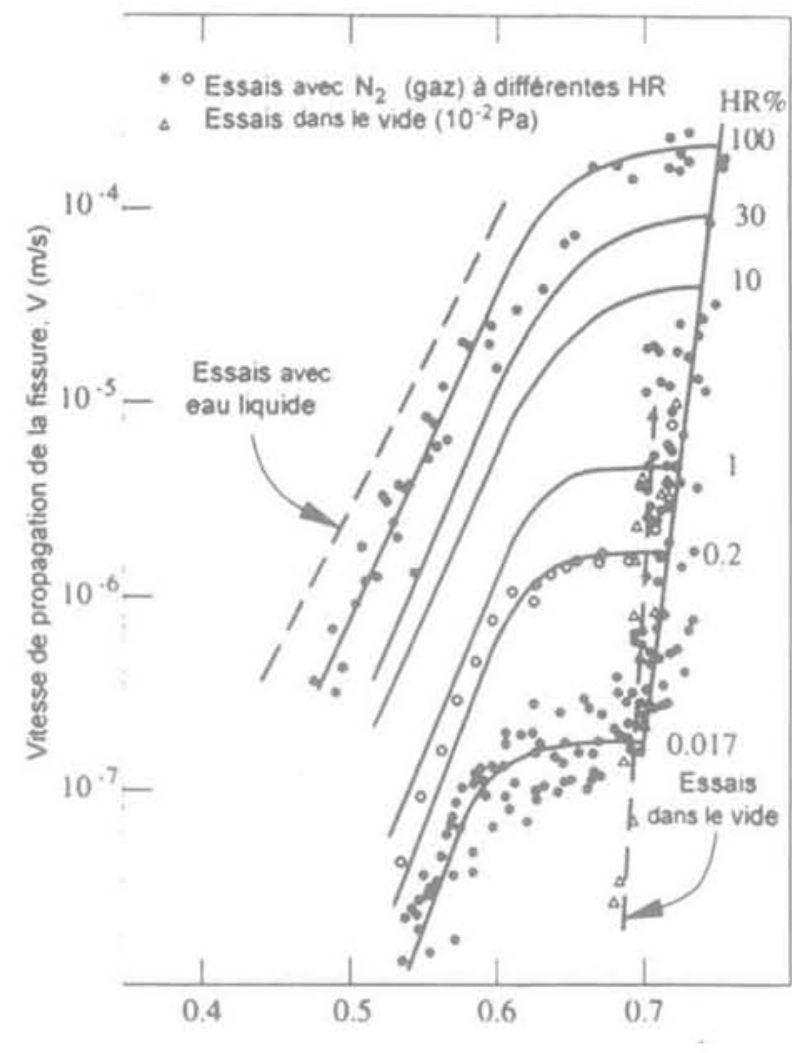

FiG. 9 Courbes expérimentales de propagation de vitesse de fissures dans le verre (Wiederhorn et al., 1980).

Experimental results of crack propagation velocity in glass (Wiecierhorn et al, 1980).

Dans le cas de l'eau et de solutions d'autres liquides ou de sels contenues dans celle-ci, l'humidité relative est une façon de décrire le potentiel chimique, ou la succion. En effet, la loi psychrométrique établit:

$$
\mathrm{RH}=\exp \left[-\frac{\mathrm{M}_{\mathrm{v}}(\mathrm{s}+\pi)}{\mathrm{RT}}\right]
$$


où $\psi=\mathrm{s}+\pi$ est la succion totale, $\mathrm{s}$ est la succion capillaire et $\pi$, la succion osmotique. $M_{y}$ est le volume molaire de l'eau.

Dans la ligne de ces idées, un précédent intéressant est fourni par les essais réalisés par Martín Viñas (1973) sur de la roche triturée. Il attribua les déformations d'effondrement de l'enrochement à l'écrasement des particules de roche sous l'action conjointe des contraintes et de l'affaiblissement des forces électrostatiques de cohésion dans les liaisons atomiques de la roche en présence de molécules dipolaires telles que celle de l'eau et choisit d'expliquer l'effondrement en utilisant la constante diélectrique comme variable fondamentale du liquide saturant les vides de l'enrochement. Il réalisa des essais d'effondrement dans un œdomètre en inondant avec différents liquides et observa la corrélation montrée sur la figure 10 entre l'effondrement et la constante diélectrique. Le travail de Martín Viñas a mis en évidence que c'est une propriété fondamentale du liquide présent dans les vides de l'enrochement qui contrôle, avec l'état de contraintes, l'effondrement. Dans les travaux d'ingénierie, c'est généralement l'eau, avec une concentration variable en sel, qui occupe partiellement les pores de la roche et les vides de l'enrochement. Dans ce cas, la variable fondamentale qui explique la vitesse de rupture des particules est l'humidité relative du gaz dans les vides de l'enrochement ou, alternativement, la succion totale de l'eau.

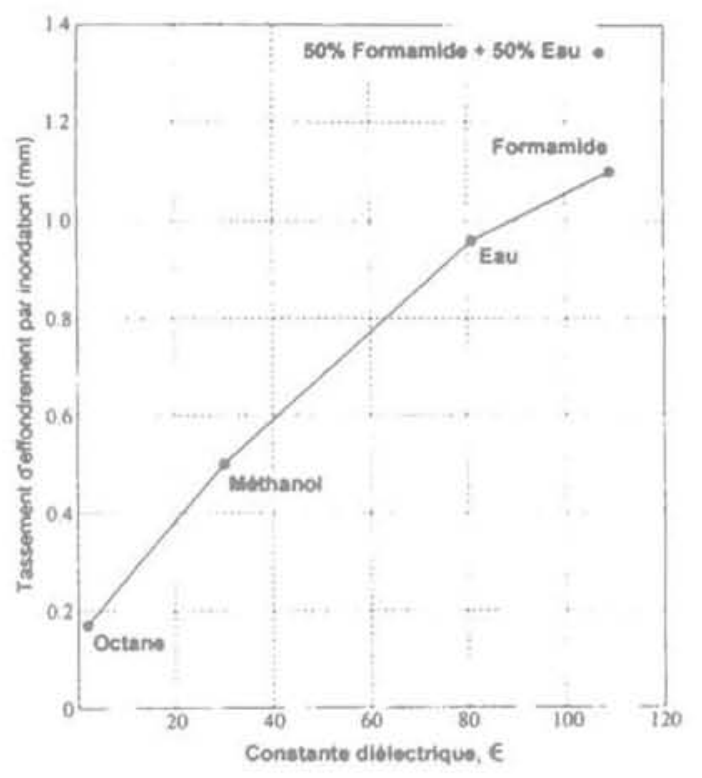

FiG.10 Corrélation expérimentale entre la déformation d'effondrement mesurée lors d'un essai œdométrique sur calcaire trituré (sous une charge verticale constante de 1,6 MPa) et la constante diélectrique des différents liquides employés pour inonder les éprouvettes (Viñas, 1973). Diamètre de l'œdomètre $=70 \mathrm{~mm}$, hauteur de l'éprouvette $=70 \mathrm{~mm}$, granulométrie entre tamis \#4 et \#10 (ASTM).

Experimental correlation between collapse deformation measured an oedometer test of crushed limestone (under a constant vertical stress of $1.6 \mathrm{MPa}$ ) and the dielectric constant of different flooding liquids (Viñas, 1973). Oedometer diameter: $70 \mathrm{~mm}$; Specimen height: $70 \mathrm{~mm}$. Grain sizes in the range ASTM \#4 and \#10.
Les données expérimentales de la figure 9 ou de l'équation (3) peuvent se synthétiser par le schéma de la figure 11 qui représente la vitesse de propagation de la rupture en fonction du facteur d'intensité des contraintes, $\mathrm{K}$, et l'humidité relative $\mathrm{RH}$. L'axe $\mathrm{K}$ est divisé en trois régions : I, II et III, limitées par deux valeurs critiques de $\mathrm{K}$ : la valeur au-dessous de laquelle la propagation de la rupture $\left(\mathrm{K}_{0}\right)$ est impossible et la valeur de ténacité $\mathrm{K}$. Les físsures dont l'état se situe dans la région $\mathrm{K}_{\mathrm{r}}<\mathrm{K}<\mathrm{K}$ se propagent à une vitesse qui dépend de $\mathrm{RH}$ et de la contrainte appliquée. Lorsque $\mathrm{K}=\mathrm{K}$, la rupture est instantanée. Dans une situation d'équilibre (sans déformation), toutes les fissures des particules de l'enrochement auront lieu dans la région I. Si l'on incrémente la charge, l'état des fissures se déplacera le long de l'axe K. Certaines atteindront la région III. Une rupture instantanée de ces particules aura lieu, provoquant une déformation instantanée. D'autres particules auront un état qui occupera la région II. Celles-ci connaitront un processus de propagation de la rupture ( $\mathrm{K}$ augmentera) jusqu'à la rupture complète. La rupture progressive de ces particules contribuera à générer une composante de déformation différée. L'augmentation de RH a deux effets : il augmente, d'une part, la vitesse de propagation de la rupture (et donc, la vitesse de déformation macrostructurale) et il étend, d'autre part, la région II à des valeurs de plus en plus petites de $\mathrm{K}_{0}$, ce qui génère de nouvelles fissures. Selon cette interprétation, l'effondrement par inondation correspond aux déformations qui se produisent à contrainte constante lorsque l'humidité relative atteint $100 \%$. Ce modèle conceptuel est schématiquement représenté sur la figure 11 . Il indique qu'un enrochement $\sec (\mathrm{RH}=0 \%)$ n'expérimentera pas de déformations différées (car dans ce cas $\mathrm{K}_{0} \equiv \mathrm{K}_{\mathrm{f}}$ ), mais que son potentiel d'effondrement est haut, dans le cas où l'humidité relative ambiante augmente suffisamment pour que les fissures les plus sollicitées soient activées.

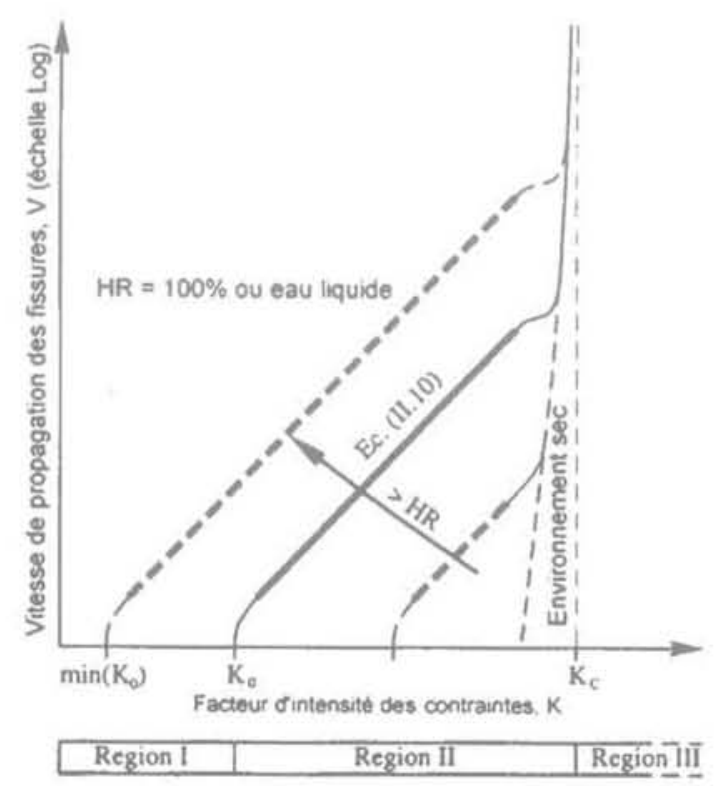

FiG.11 Représentation schématique des courbes de propagation subcritique des fissures pour le modèle conceptuel proposé.

Schematic representation of subcritical crack propagation for the conceptual model proposed. 
On a décrit un mécanisme de déformation des enrochements lié à la rupture des particules. Il coexiste sûrement avec des autres mécanismes, tels que le simple réarrangement de particules, communs dans les sols. Un modèle rhéologique macroscopique se doit d'intégrer les différents mécanismes qui contribuent à la déformation sur la base de données expérimentales du comportement d'enrochement en réponse à certains cheminements de contraintes. Les idées antérieures suggèrent qu'une variable fondamentale de ces trajectoires est l'humidité relative du milieu ambiant. Pour valider cette idée, on a planifié et réalisé une série d'essais cedométriques sur roche triturée avec contrôle de l'humidité relative.

\section{3}

\section{Essais œdométriques avec contrôle d'humidité relative}

Les essais ont été conduits sur une ardoise quartzique cambrique originaire d'un affleurement proche de l'emplacement du futur barrage sur le fleuve Pancrudo en Aragon (Fig. 12). A partir de particules extraites à la pelle rétro, on a obtenu par trituration un matériau granulaire de taille maximale des grains proche de $4 \mathrm{~cm}$ (Fig. 13). Sur la figure 14, la courbe granulométrique du matériau est représentée. Les autres propriétés de la roche sont : une résistance à la compression simple

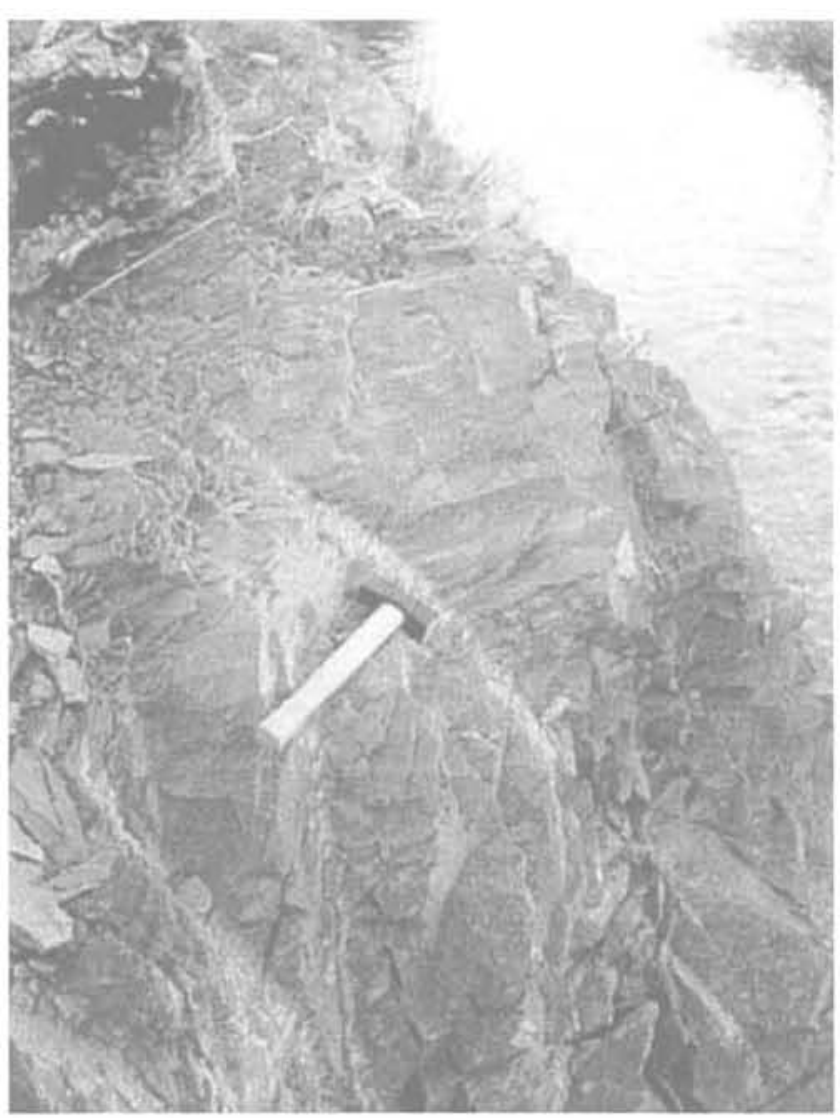

FIG.12 Photographie de l'affleurement d'ardoise du Pancrudo, à l'endroit d'obtention du matériau testé.

Pancrudo shale outcrop. égale à 20,5 MPa (14,2-31,9 MPa) déterminée sur des échantillons équilibrés a l'humidité relative du laboratoire $(\mathrm{RH} \cong 60 \%$ ), un poids spécifique relatif égal à 2,754 et une porosité de $8 \%(6,3-11,8 \%)$. La courbe de rétention des particules de roche, mesurée avec différentes techniques d'application de la succion (technique tensiométrique pour les succions très basses ; de surpression d'air pour des succions moyennes et de contrôle de l'humidité relative pour les succions hautes) est indiquée sur la figure 15. La teneur en eau à saturation des particules de roche est d'environ $2,5 \%$. La saturation du matériau granulaire se produit à une teneur en eau d'environ $20 \%$. Les échantillons testés ont été compactés à l'aide d'un marteau au travers d'une base interposée qui évite un coup direct sur les particules de roche. Les échantillons ont été compactés directement dans l'anneau œdométrique en quatre couches ayant reçu chacune une énergie de 600-700 Joules/l (proche de celle correspondant à l'essai du Proctor normal: 584,3 Joules/1). Après compactage, l'indice des vides était égal à $0,55 \pm 0,03$. Avant compactage, la teneur en eau des échantillons s'était équilibrée avec l'humidité ambiante du laboratoire $\left(30 \% \mathrm{HR}\right.$ à $\left.22^{\circ} \mathrm{C}\right)$. La teneur en eau initiale était donc basse, inférieure à $1 \%$.

On a construit deux oedomètres à humidité relative contrôlée destinés à recevoir des échantillons de $300 \mathrm{~mm}$ de diamètre et de $200 \mathrm{~mm}$ de hauteur. La procédure de contrôle de l'HR est indiquée sur la figure 16 : on fait circuler l'air contenant un pourcentage en vapeur d'eau obtenu par barbotage dans une solution
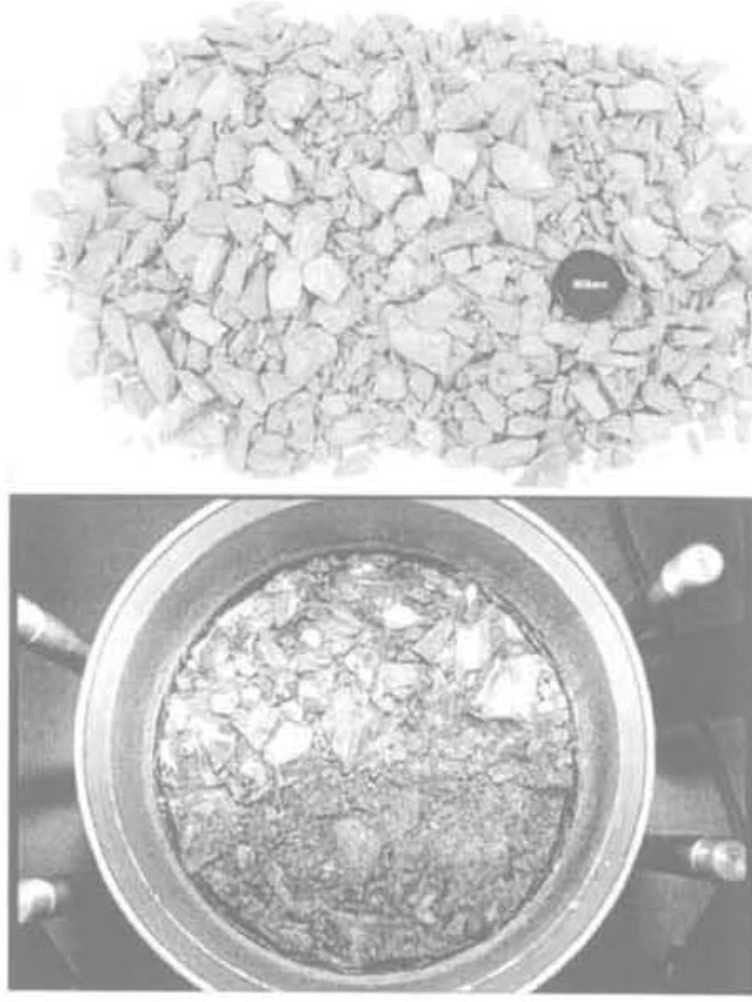

(a)

FIG. 13

Matériel testé. (a) Avant le compactage. (b) Éprouvette compactée dans I'anneau œedométrique.

Tested material. (a) Before compaction. (b) Compacted specimen inside the oedometer cell. 


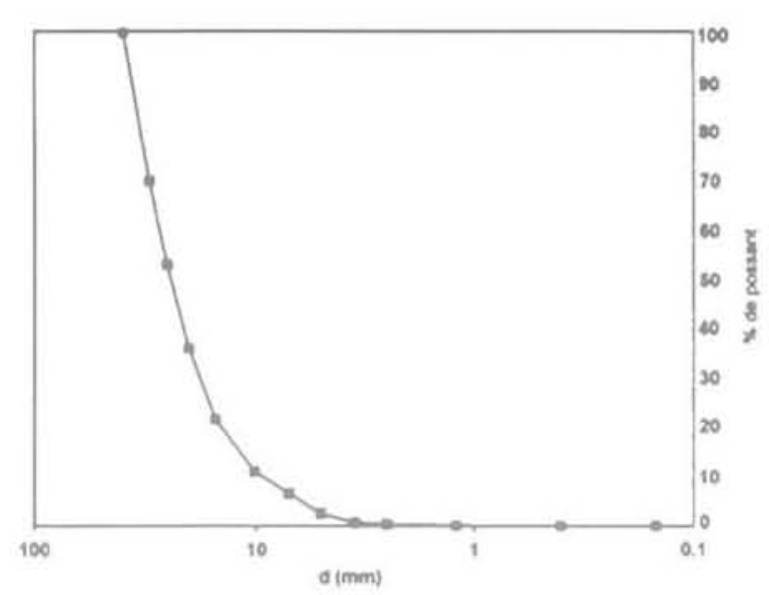

FiG.14 Granulométrie d'enrochement du matériau testé (avant le compactage). Ardoise du Pancrudo triturẻe. Taille maximale $=40 \mathrm{~mm}$, taille minimale $=0,4 \mathrm{~mm}, \mathrm{Cu}=2,9$.

Grain size distribution of tested gravel before compaction. Crushed Pancrudo shale. Maximum and minimum sizes $=40$ and $0.4 \mathrm{~mm}$, uniformity coefficient, $\mathrm{Cu}=2.9$

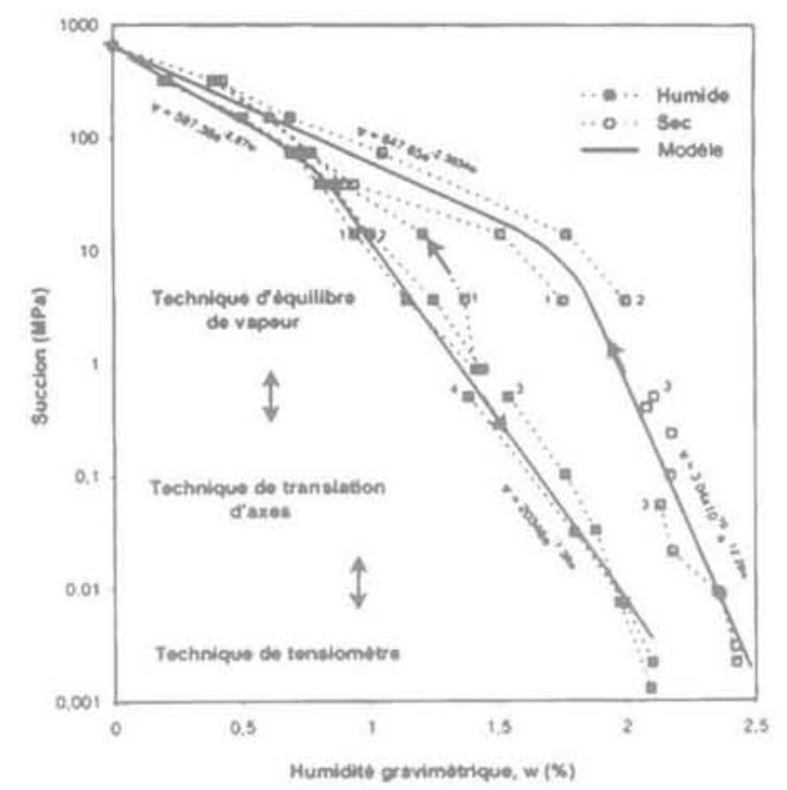

คG. 15 Courbe de rétention de l'ardoise du Pancrudo. Données expérimentales et formules mathématiques par rapport aux branches d'humidification et de séchage. Les chiffres identifient les échantillons essayés.

Water retention curve of the Pancrudo shale. Experimental data and mathematical expressions interpolated. Numbers identify samples tested.

saline à concentration fixée. Après une période d'équilibre, l'humidité relative dans les vides de l'enrochement correspond à un HR en équilibre thermodynamique avec la solution saline de contrôle. Un hygromètre placé dans le circuit à proximité de l'échantillon fournit une mesure directe du HR atteint. La vapeur d'eau se déplace au travers des (grands) vides de l'enrochement à l'aide des phénomènes advectifs.
La migration de la vapeur d'eau à l'intérieur des pores de la roche sera par contre contrôlée par des phénomènes de diffusion (Fig. 16). Un enrochement est en fait un matériau avec une double porosité très marquée. Cela permet de parler d'une part du HR des grands vides et d'une autre part du HR des pores de la roche, généralement différent. Entre les deux, s'établiront des mécanismes locaux de transfert qui seront examinés dans la dernière section de cet article. Dans cette section, on présentera des résultats correspondant à la situation d'équilibre (égalité) entre les deux humidités relatives.

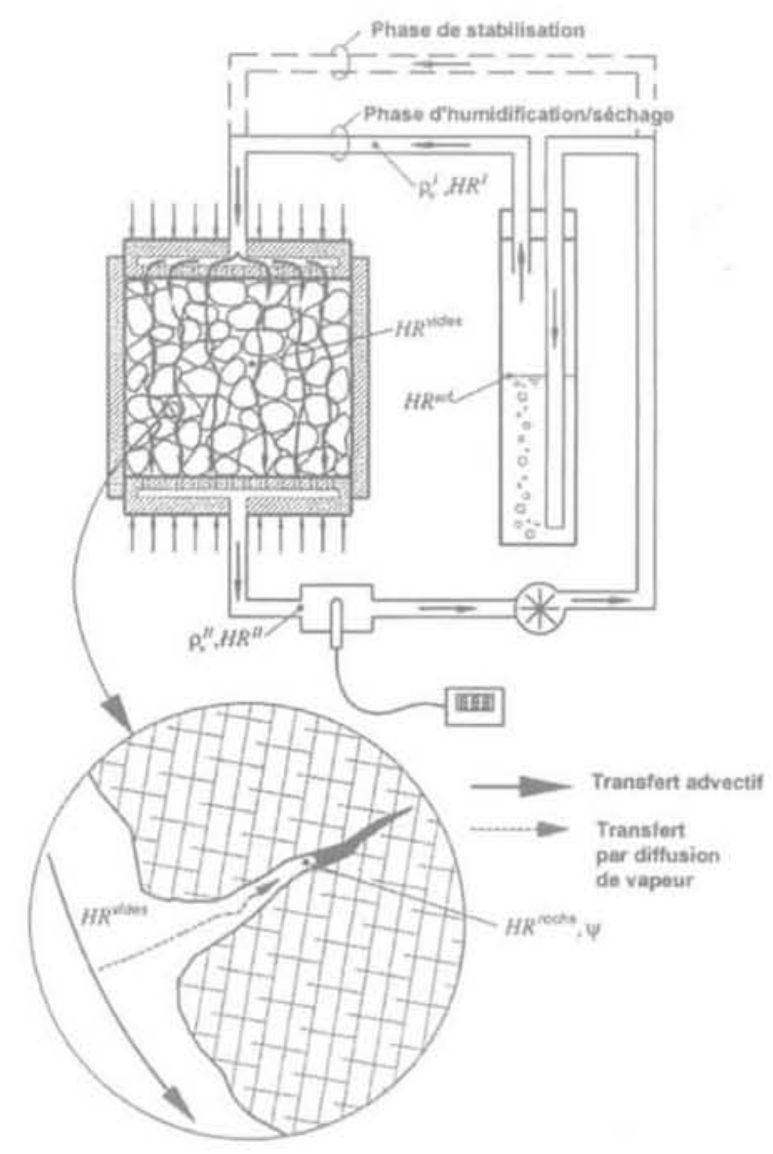

FGG 16 Schéma du système de contrôle d'HR. Nomen. clature des variables employées dans le modèle analytique.

Scheme of RH controlled oedometer cell.

Les deux équipements construits sont illustrés sur les figures 17 et 18. La taille des échantillons est la même dans les deux oedomètres. Le premier équipement construit (Fig. 17) est un oedomètre de type Rowe en laiton. Les résultats présentés ici correspondent à cette cellule. Dans le deuxième (le schéma est montré sur la figure 19), on a introduit des améliorations : possibilité d'augmenter la contrainte verticale jusqu'à une valeur de $27 \mathrm{MPa}$, un meilleur contrôle du HR, une mesure des charges verticales sur l'appui inférieur, qui permet de déterminer la friction moyenne sur l'anneau de confinement ainsi qu'un dispositif de mesure des contraintes horizontales. 


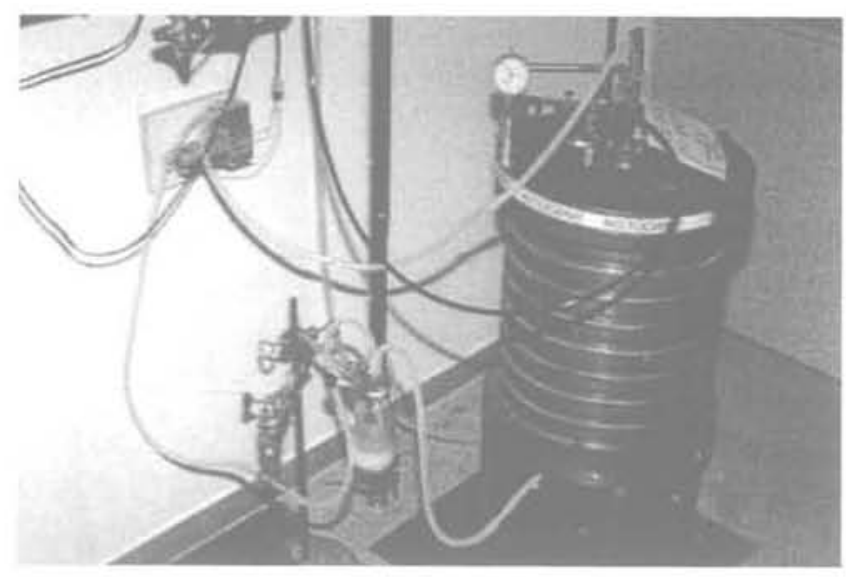

FiG.17 Photographie de l'œdomètre Rowe (équipement I) employé dans le programme d'essais.

Rowe type oedometer (I) used in the first series of tests performed.

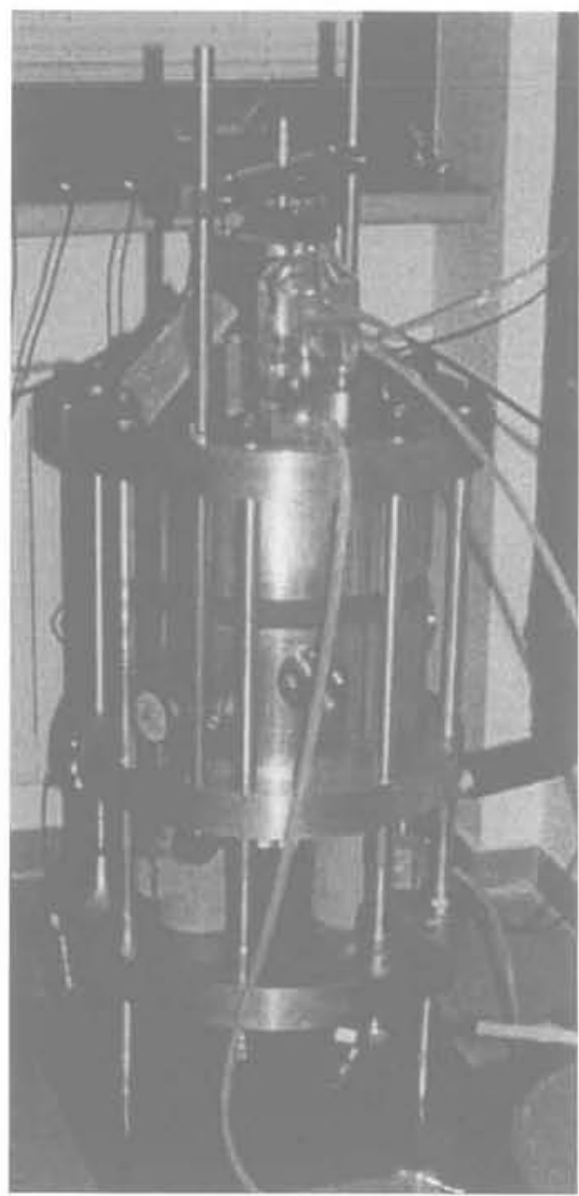

FIG. is Photographie de I'œdomètre II, développé pour l'essai de matériaux d'enrochements avec contrôle de HR. Diamètre de l'éprouvette $=300 \mathrm{~mm}$. Hauteur de l'éprouvette $=$ $200 \mathrm{~mm}$. Contrainte verticale maximale $=$ $2,8 \mathrm{MPa}$. L'équipement permet la mesure de la contrainte latérale et du frottement entre l'éprouvette et la paroi de l'oedomètre.

$\mathrm{RH}$ controlled oedometer cell (II) used in the second series of test performed. Sample diameter $=300 \mathrm{~mm}$. Sample height $=200 \mathrm{~mm}$. Maximum vertical stress $=2.8 \mathrm{MPa}$. Lateral stresses and side friction can be measured.

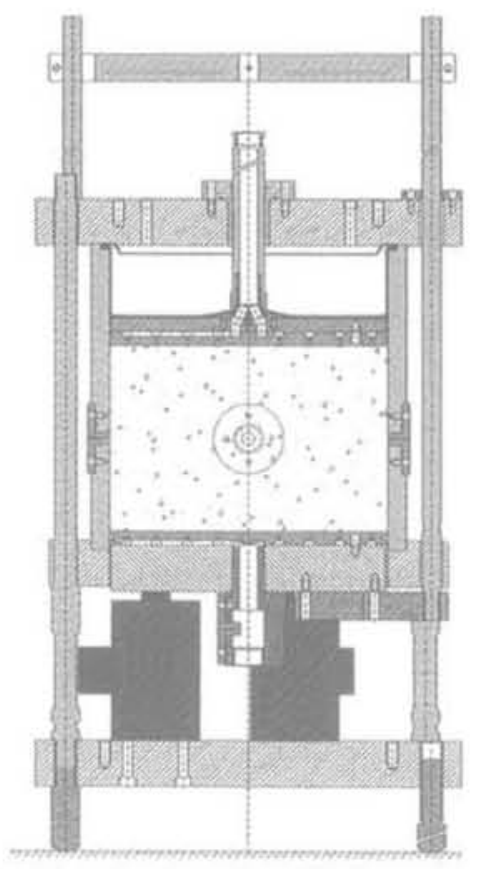

FIG 19 Schéma de l'œdomètre pour enrochements II. Scheme of RH controlled oedometer cell (II).

Les résultats des cinq essais de compression effectués dans le premier oedomètre sont indiqués sur la figure 20. Sur la figure 20b, on a indiqué les trajectoires des contraintes appliquées dans un plan : succion totale vs. contrainte verticale. Les déformations mesurées dans ces essais sont représentées sur la figure 20a. La charge a été appliquée par incréments de 24 heures. Les données de la figure 20 correspondent à $t=$ $1000 \mathrm{~min}$.

L'analyse des courbes de déformation en fonction du temps après l'immersion à contrainte verticale constante (c'est le cas de l'essai 3, sous une contrainte de $0,6 \mathrm{MPa}$ ) montre que l'effondrement est associé à une accélération des déformations différées. Dans l'essai 4, la trajectoire de l'essai 3 est répétée, mais cette fois l'humidification de l'échantillon sous $\sigma_{\mathrm{v}}=0,60 \mathrm{MPa}$ se fait en augmentant le $\mathrm{RH}$ par étapes (c'est-à-dire en réduisant par étapes la succion totale). L'effondrement final, atteint lorsqu'on a $\mathrm{RH}=100 \%$, est similaire à celui de l'échantillon 3. Pourtant dans l'essai 4, l'échantillon n'a pas été inondé et les vides entre particules ne sont pas remplis de liquide.

L'ensemble des essais peut être synthétisé en une série de conclusions :

Les déformations induites par une augmentation de charge ont deux composantes : instantanées qui diminuent avec la contrainte de confinement et qui ne semblent pas être affectées par la teneur en eau; et différées, qui augmentent avec l'intensité de la contrainte appliquée et la teneur en eau initiale. Les déformations différées semblent être négligeables sous une contrainte de confinement, qui dans le cas des échantillons testés, est d'environ $0,4 \mathrm{Mpa}$; 

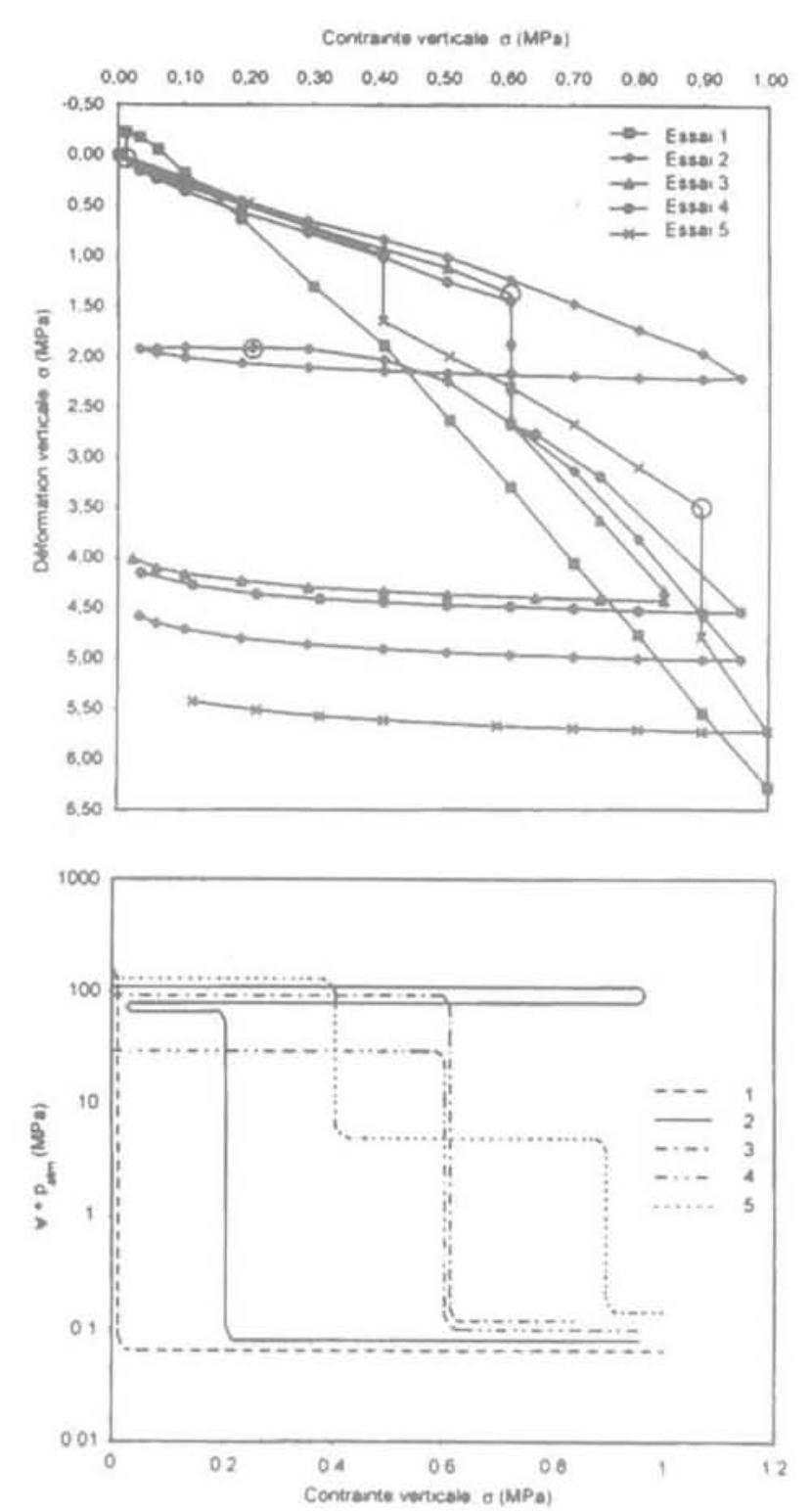

FIG. 20 (a) Résultats des essais œdométriques sur enrochements, programme 1. Matériau: ardoise du Pancrudo. Contrainte verticale à échelle naturelle. Les cercles indiquent l'inondation de l'éprouvette. (b) Trajectoires suivies dans les essais cedométriques dans l'espace contrainte verticale-succion totale. (a) Oedometer tests performed in experimental program $\mathrm{I}$. Vertical stress is shown in natural scale. Circles indicate specimen flooding. (b) Stress paths followed by tests in a vertical stress total suction plane.

Il y a une courbe de compression unique (linéaire dans le plan contrainte-déformation) pour chaque humidité relative (ou succion totale). Si $\psi$ augmente, le matériau se rigidifie;

A chaque instant du processus de charge, on peut définir un domaine élastique ;

L'effet de l'humidification dépend de la contrainte de confinement. On ne mesure pas d'effondrement en dessous d'une certaine contrainte de confinement.

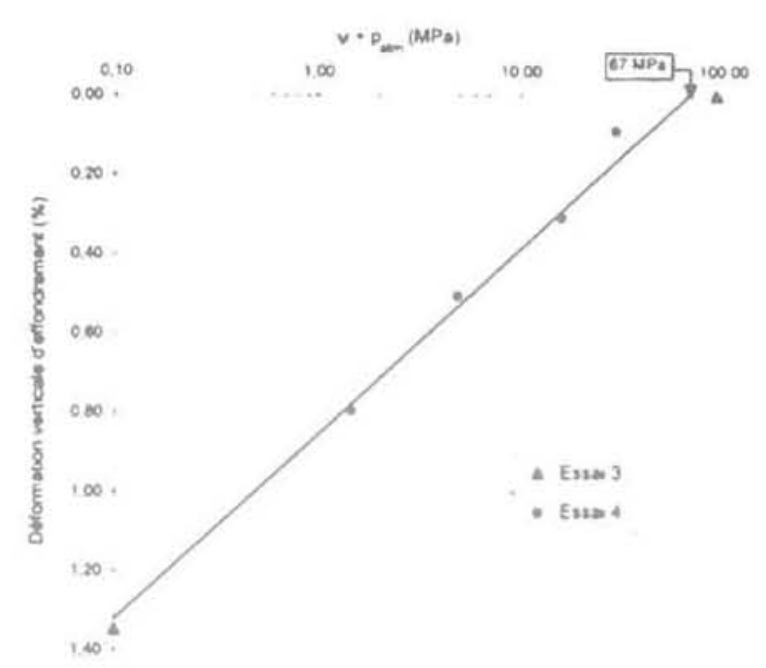

FG. 21 Corrélation entre déformation d'effondrement et succion totale à partir des mesures obtenues dans les essais 3 et 4 .

Correlation between collapse deformation and fotal suction. Data from tests 3 and 4 .

\section{Modèle rhéologique}

A partir des observations expérimentales et des implications du modèle conceptuel décrit auparavant, on a formulé un modèle élastoplastique pour le comportement des enrochements. La formulation a trois étapes : dans la première, on considère des états isotropes de contrainte et déformation et on introduit l'influence de l'eau. Dans la deuxième, on combine le modèle de compressibilité isotrope avec des états de cisaillement dans l'espace triaxial des contraintes, Finalement, on généralise le modèle en employant un critère de rupture du type Von Mises. Dans Oldecop et Alonso (2001), il y a une description plus détaillée du modèle de compressibilité.

Conformément aux expériences, on suppose que la relation contrainte (moyenne)-déformation est linéaire. La pente de cette ligne (taux de compression) dépend de la succion totale. Si la contrainte moyenne appliquée, $\mathrm{p}$, est inférieure à une valeur seuil, $\mathrm{p}_{\mathrm{v}}$, le taux de compression est constant. Lorsque $p<p_{y}$ il n'y a pas de déformations différées et le matériau granulaire réagit seulement par des déformations instantanées. Le paramètre $p_{y}$ est interprété comme étant la contrainte de confinement qui marque le début de la rupture de particule. Il sera donc lié au facteur d'intensité des contraintes $\mathrm{K}_{0}$ défini auparavant. C'est-à-dire, si $\mathrm{p}<\mathrm{p}_{\mathrm{y}}$ les fissures des particules de roche sont dans la régionf (Fig. 11). Dans ce cas la déformation de l'échantillon est seulement due au réarrangement des particules:

$$
\text { Si } p<p_{y} d \varepsilon=\lambda^{\prime} d p
$$


Si $p \geq p_{y}$ des déformations instantanées et différées ont lieu. Les deuxièmes dépendent de la succion $\psi$ selon:

Si $p \geq p_{y} d \varepsilon=\left[\lambda+\lambda^{d}(\psi)\right] d p$

où $\left(\lambda+\lambda^{d}(\psi)\right)$ est la pente de la courbe de compression vierge lorsque $\mathrm{p}>\mathrm{p}_{y^{\prime}}$

Lors de trajectoires de déchargement et rechargement (élastiques):

$$
\mathrm{d} \varepsilon^{\epsilon}=\kappa \mathrm{d} \sigma
$$

où, d'après les essais, $\kappa$ est supposée indépendante de l'humidité relative. On introduit de plus une déformation de gonflement lorsque la succion diminue dans le domaine élastique:

$$
\mathrm{d} \varepsilon^{\psi}=\kappa_{\psi} \frac{\mathrm{d} \psi}{\left(\psi+\mathrm{P}_{\text {atm }}\right)}
$$

Afin de simplifier le modèle, le taux d'expansion/ rétraction causé par les variations de succion, $\kappa_{\psi}$, est supposé indépendant de la succion.

Avec ces hypothèses on peut obterir la surface de charge suivante dans l'espace (p, $\psi$ ):

$F(p, \psi)=p_{0}\left[\lambda+\lambda^{d}(\psi)-\kappa\right]-p_{y} \lambda^{d}(\psi)-p_{0}^{\prime}\left(\lambda^{\dagger}-\kappa\right)=0$

où $p_{0}$ est le seuil de plasticité à la succion $\psi$ et $p_{0}^{*}$, est le seuil de plasticité pour des états très secs, choisi comme le paramètre d'écrouissage.

L'équation (9) est valide si $p>p_{y^{\prime}}$. Si $p<p_{y^{\prime}}$ la surface de charge s'écrit simplement:

$$
\mathrm{F}(\mathrm{p}, \psi)=\mathrm{p}_{0}-\mathrm{p}_{0}^{\prime}
$$

On propose comme loi d'écrouissage une simple loi de compression isotrope :

$$
\mathrm{dp}_{0}^{*}=\mathrm{d} \varepsilon^{\mathrm{p} /}\left(\lambda^{\prime}-\kappa\right)
$$

où $\varepsilon^{p}$ est la déformation volumique plastique.

Conformément aux résultats expérimentaux, le coefficient de compressibilité $\lambda^{d}(\psi)$ s'exprime en fonction de la succion totale de la façon suivante :

$\lambda^{d}(\psi)=\lambda_{o}^{d}-\alpha_{\psi} \ln \left(\frac{\psi+p_{\text {atm }}}{p_{\text {atm }}}\right) ; \lambda^{d} \geq 0$

oủ $\lambda_{0}^{d}$ et $\alpha_{\psi}$ sont des paramètres du modèle et $\lambda_{0}^{d}$ est le taux de compressibilité du matériau saturé. Pendant le séchage du matériau, $\psi$ augmente et la compressibilité diminue jusqu'à atteindre un " état très $\sec$ ». Dans ce cas, le taux de compressibilité est $\lambda^{\prime}$ (puisque $\lambda^{0}=0$ ). Conformément aux données expérimentales présentées, pour l'ardoise de Pancrudo (Fig. 21) $\psi=67 \mathrm{MPa}$ définit l'état très sec.

Si l'équation (12) est introduite dans l'équation (9), une expression explicite de la surface de charge est obtenue. La figure 22 montre les courbes de charges pour des valeurs différentes du paramètre d'écrouissage $p_{0}^{*}$ : Les zones dans lesquelles le comportement de l'enrochement ne dépend pas de l'action de l'eau sont indiquées en gris. Elles correspondent à des états de contrainte moyenne inférieure à $\mathrm{p}_{\mathrm{y}}$ ainsi qu'à des états très secs.

Ce modèle isotrope peut s'étendre facilement à un état de contrainte triaxial moyennant un modèle de référence qui introduit l'effet des contraintes de cisaillement. Par simplicité, le modèle de Cam-Clay

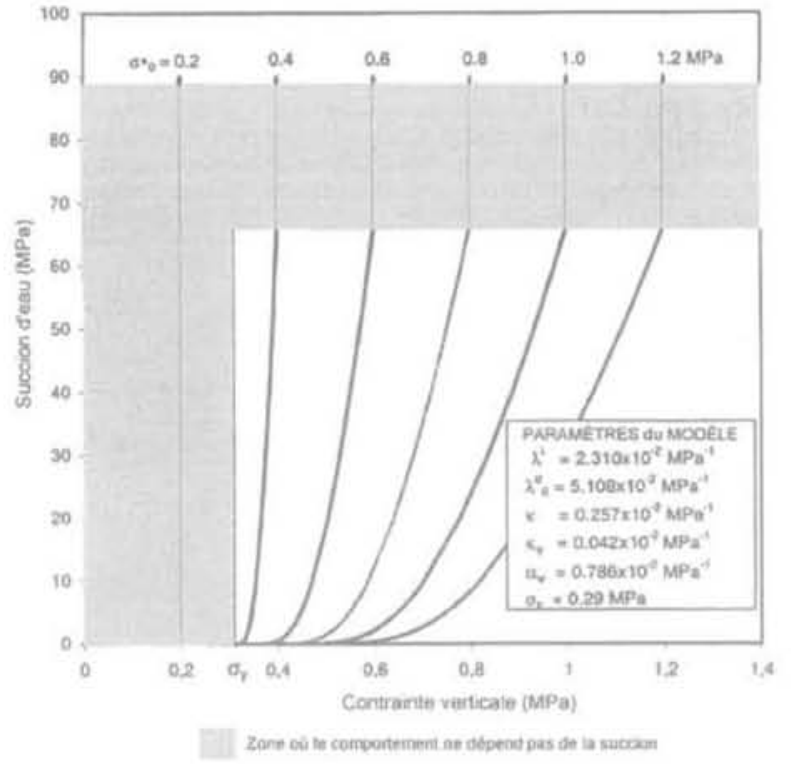

HQ. 22 Courbes de charge plastique. Yield curves.

modifié est choisi et la nouvelle surface de charge s'écrit:

$F(p, q)=q^{2}-M^{2} p\left(p_{0}-p\right)=0$

où q est la contrainte déviatorique, $\mathrm{p}_{0}$ le seuil de plasticité en compression isotrope et $\mathrm{M}$ la pente de la ligne d'état critique (paramètre du modèle). Il serait même possible d'étendre le modèle isotrope à d'autres types de modèles dans le plan déviatorique.

A partir de l'expression de surface de charge (9), la valeur de $\mathrm{p}_{0} \mathrm{~s}^{\prime}$ obtient par:

$$
p_{0}=\left[p_{0}^{*}\left(\lambda^{\prime}-\kappa\right)+p_{y} \lambda^{d}\right] /\left(\lambda^{\prime}+\lambda^{d}-\kappa\right)
$$

Les équations (12), (13) et (14) définissent la surface de charge dans le plan (p, q, $\psi$ ).

Une version généralisée de (13), quí incorpore le critère de rupture de Von Mises, est (Gens et Potts, 1988):

$$
F(p, J)=3 J^{2}-M^{2} p\left(p-p_{0}\right)
$$

où $\mathrm{J}^{2}$ est le deuxième invariant du tenseur de contraintes déviatoriques. A partir des équations (13) et (14), un potentiel plastique prédisant de manière adéquate le coefficient de poussée des terres au repos $\mathrm{K}_{0}$ (Ohmaki, 1982) peut être construit. Il a la forme:

$$
G(p, J)=3 J^{2}-\alpha M^{2} p\left(p-p_{0}\right)
$$

où $\alpha$ est relié à la valeur de $\mathrm{K}_{0}$.

Les équations antérieures définissent le modèle de comportement des enrochements. Dont les paramètres sont: $p_{v}, \lambda, \lambda_{v}^{d}, \alpha_{v}, \kappa, \kappa_{v}, M$ et $\alpha$.

Les paramètres définis ont une signification physique, ce qui permet leur obtention facile à partir des données expérimentales. Les essais 1, 2 et 3 conduisent à identifier les valeurs suivantes dans le cas de l'ardoise compactée:

$\mathrm{p}_{\mathrm{y}}=0,29 \mathrm{MPa}, \lambda^{i}=2,3 \times 10^{-2} \mathrm{MPa}, \lambda_{0}{ }^{\mathrm{d}}=5,1 \times 10^{-2} \mathrm{MPa}$, $\alpha_{w}=0,78 \times 10^{-2} \mathrm{MPa}^{-1}, \kappa=0,26 \times 10^{-2} \mathrm{MPa}^{-1}$, $\kappa_{\psi}=0,04 \times 10^{-2} \mathrm{MPa}^{-1}$.

Avec ces valeurs, les essais 4 et 5 ont été simulés. Le résultat de la comparaison entre les résultats de l'Essai 5 et ceux du modèle est indiqué sur la figure 23. L'effon- 
drement partiel mesuré sous une contrainte verticale de 0,4 MPa n'est pas bien reproduit par le modèle mais le comportement général est représenté de manière satisfaisante.

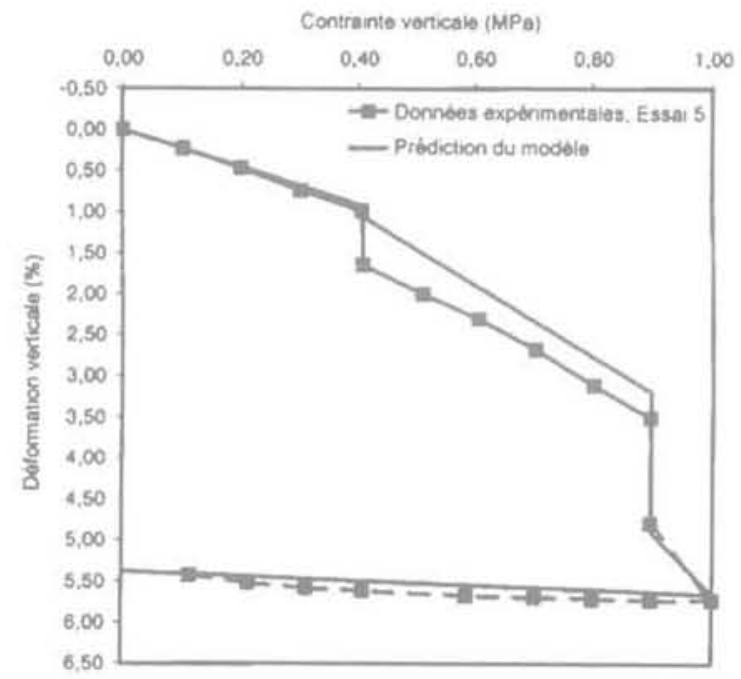

716. 2a Essai 5. Comparaison modèle-expérience. Test 5. Comparison between experimental results and model calculations.

\section{5}

\section{Écoulement et déformation dans un enrochement}

La haute perméabilité d'un barrage ou d'un remblai en enrochement fait que la condition d'écoulements saturés est en pratique très improbable. La recharge amont d'un barrage zoné est inondée lorsque le niveau de la retenue augmente, tandis que la recharge aval est sujette à l'infiltration causée par la pluie et à l'évaporation. Ces dernières conditions sont celles qui prévalent dans les remblais en enrochements des voies de communication. Les grands vides du matériau d'enrochement resteront essentiellement pleins d'air, avec des quantités variables de vapeur d'eau, pendant la vie utile de ces structures. Comme illustré sur la figure 24 , l'écoulement en phase liquide a tendance à ètre un écoulement à surface libre, à l'intérieur des vides de l'enrochement, en se déplaçant en surface des particules. Les particules de roche n'étant pas complètement saturés, l'eau peut y pénétrer au travers de la porosité connectée.

Une autre voie de pénétration peut être les fissures présentes dans les particules. Les plus significatives d'entre elles sont celles qui existent dans les zones proches des contacts fortement comprimés.

Dans ce système complexe de transfert d'eau sous forme liquide et vapeur, on peut suggérer un modèle d'écoulement basé sur l'existence de deux états d'énergie de l'eau dans l'enrochement: l'énergie associée aux grands vides et celle qui correspond à l'intérieur des particules ou des particules poreuses. Les deux cas ont été représentés sur la figure 16. Le premier contrôlera

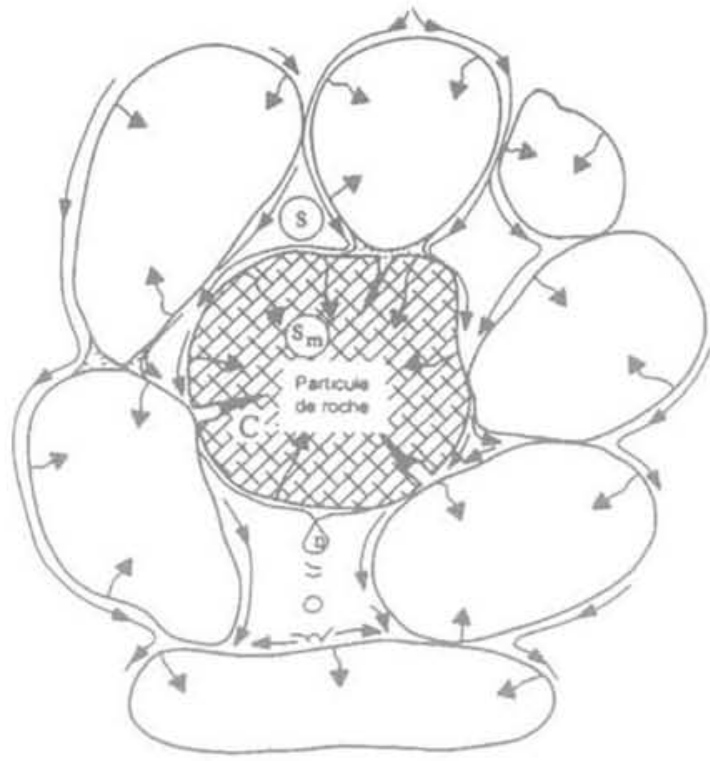

FiG, 24 Schéma de l'écoulement d'eau dans un enrochement.

Elow regime in a rockfill.

l'écoulement d'eau libre, qui sera emmagasinée aux contacts entre particules par les phénomènes capillaires, et en surface des particules de roche. Les mécanismes de transfert d'eau liquide dans ces conditions ont été analysés dans Gili (1988). On se doit toutefois de les simplifier afin de les réduire à un transfert du genre Darcy généralisé, tel qu'habituellement employé dans des sols non saturés. Cela conduit à définir un coefficient de perméabilité globale qui dépend de la succion ou de l'énergie totale propre des grands vides. Cette eau libre ou vapeur régnant dans les grands vides approvisionne en eau les pores et les fissures de la roche. L'écoulement local, qui peut se considérer comme un terme de puits ou source, dépendra dans une première approche de la différence entre la succion dans les grands vides $\psi$ et celle dans les pores ou fissures $\psi_{m}$. Ainsi, une formulation simple de l'écoulement entre les deux domaines de porosités s'écrit:

$$
q=-\frac{d w^{m}}{d t}=-\rho_{w} \alpha^{\prime}\left(\psi-\psi_{m}\right)
$$

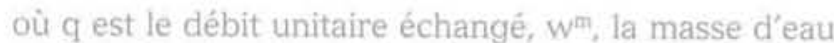
"microstructurale » (c'est-à-dire, localisée dans les vides ou fissures des particules de roches) et $\alpha^{\prime}$, un coefficient de transfert. Si l'on désigne par $\phi$ la porosité des particules de roche, la vitesse d'échange de la masse microstructurale d'eau peut se définir comme:

$$
\frac{d w^{m}}{d t}=\rho(1-\phi) \phi_{m} \frac{d S_{1}^{m}}{d t}
$$

où $\mathrm{S}_{1}$ m est le degré de saturation microstructural et $\phi$ est la porosité macrostructurale (grands vides) de l'enrochement. Les résultats de calculs présentés plus en avant dans le texte considèrent le coefficient $\alpha=\alpha^{\prime} / \phi_{j}$ $(\mathrm{MPa} \mathrm{s})^{-1}$. 
On a de plus besoin de définir la courbe de rétention d'eau dans les pores de la roche. Elle a été déterminée dans le cas de l'ardoise cambrienne du Pancrudo et représentée par l'expression suivante:

$$
S_{1}^{m}=\exp \left(-\psi_{m} / \psi_{0}\right)
$$

où $\psi_{0}$ est une succion de référence (paramètre du modèle).

On peut maintenant définir les équations de conservation du problème couplé écoulement-déformation :

\section{Conservation de la masse d'eau}

$$
\frac{\partial}{\partial t}\left(\theta_{1}^{w} S_{i} \phi+\theta_{g}^{w} S_{g} \phi\right)+\nabla \cdot\left(j_{1}^{w}+j_{g}^{w}\right)=f^{w}=q
$$

$\phi$ est la porosité macrostructurale de l'enrochement, $\theta^{\mathrm{w}}$ et $\theta$ w sont les masses d'eau par unité de volume de liquide et gaz respectivement, $S_{g}$ et $S_{1}\left(S_{g}=1-S_{1}\right)$ sont les degrés de saturation en gaz et en liquide et $j{ }^{w}, j_{a}{ }^{w}$ sont les flux d'eau (advectifs et diffusifs) dans les phases liquide et gazeuse. Le terme f $^{\text {w }}$ décrit l'apport extérieur en eau qui, dans ce cas, est représenté par le terme local de puits ou source définis par l'équation (17).

\section{Équilibre des forces}

L'équilibre des forces peut s'exprimer en fonction des contraintes totales $\sigma$ et des forces massiques b par:

$$
\nabla \cdot \sigma+\mathrm{b}=0
$$

Les composantes des forces d'origine capillaire sont ici négligeables et les formulations employées dans des sols non saturés ne sont donc pas nécessaires (Alonso et al., 1988).

Les équations (17) et (21) peuvent se résoudre en introduisant les relations rhéologiques appropriées et les restrictions d'équilibre local (Olivella et al., 1994, 1996). Dans le cas des enrochements, l'information additionnelle nécessaire est la suivante: équation d'écoulement de l'eau liquide (loi de Darcy généralisée); équation de diffusion de la vapeur d'eau (loi de Fick) et courbe de rétention du milieu granulaire.

Dans le modèle développé, la déformation des particules de roche n'est pas considérée. Seule la déformation de l'enrochement, associée à la rupture des particules, est représentée par le modẻle rhéologique décrit auparavant. Ce modèle établit une relation incrémentale entre contraintes et déformations du type:

$$
\mathrm{d} \sigma=\mathrm{D}_{\text {ep }}\left(\mathrm{d} \varepsilon-\mathrm{d} \varepsilon_{\mathrm{o}}\left(\psi_{\mathrm{m}}\right)\right)
$$

où $\mathrm{D}_{\text {e }}$ est la matrice élastoplastique tangente et d $\varepsilon_{p}$ sont les déformations associées aux changements de teneur en eau ou de succion microstructurale (à l'intérieur des particules de roche). Les expressions pour $D_{\text {ep }}$ et $d \varepsilon_{0}$ dérivent des relations rhéologiques auparavant présentées, en considérant que la succion qui intervient dans les équations est la succion existante dans les particules de roche.

La formulation présentée a été intégrée dans un programme général d'éléments finis pour la résolution de problèmes thermohydromécaniques (CODE_BRIGHT, Olivella et al., 1994, 1996) aux conditions avec limites. Premièrement, l'essai œdométrique 2 a été simulé. L'échantillon a été discrétisé moyennant 10 éléments à forte perméabilité macrostructurale, ce qui garanti un transfert rapide des succions depuis le contour vers l'intérieur de l'échantillon. Sur la figure 25, on indique le cheminement de contraintes et de succion imposé pendant l'essai. L'échantillon est chargé initialement, puis déchargé et rechargé jusquu'à $0,2 \mathrm{MPa}$ à une succion constante de $92 \mathrm{MPa}$. Ensuite, la teneur en eau est augmentée jusqu'à saturation sous contrainte verticale constante. Finalement, un cycle de chargement et déchargement à succion nulle est appliqué. Dans la simulation, on a employé les paramètres du modèle rhéologique présenté auparavant.
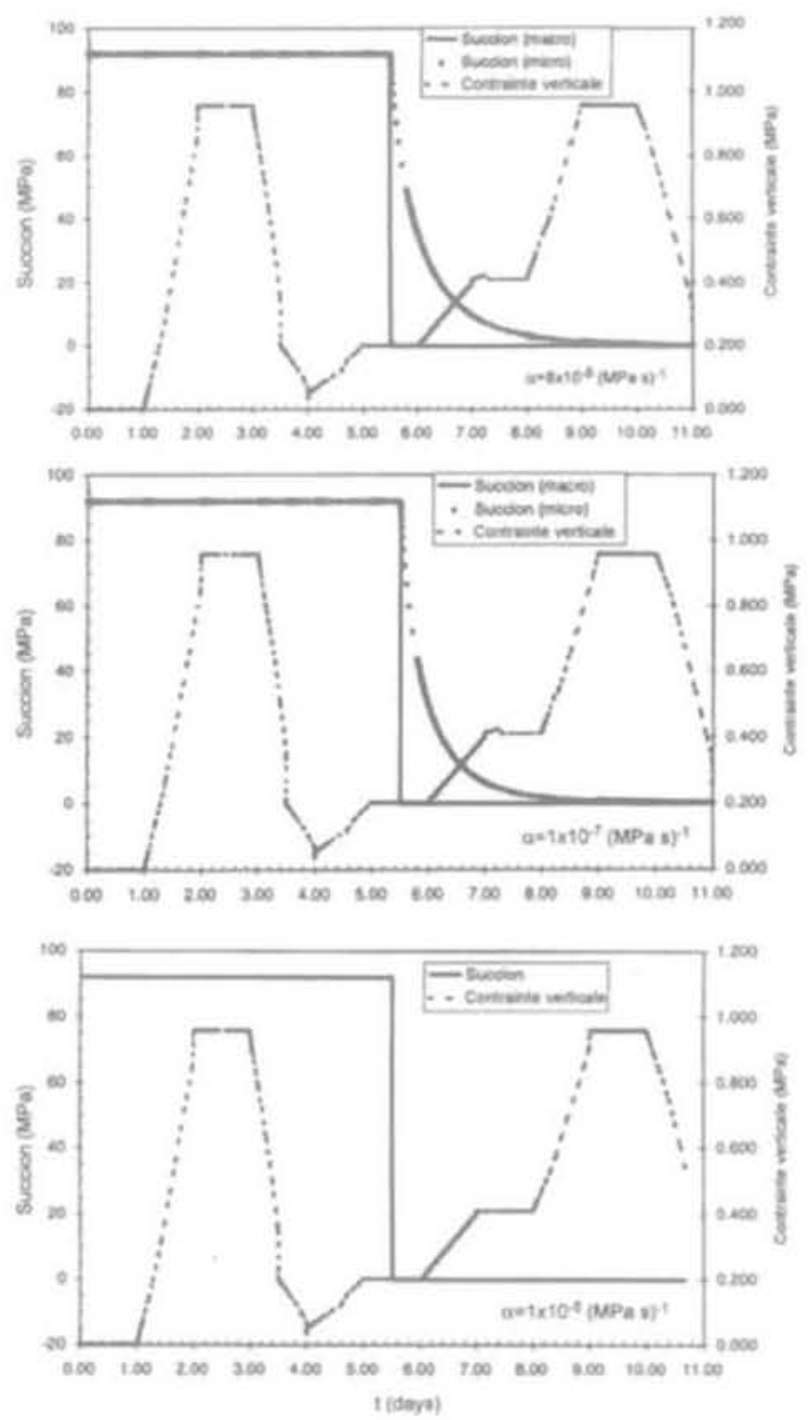

FIG. 25 Essai 2. Histoire des contraintes et succions. Prédictions du modèle pour les trois valeurs du coefficient de transfert $\alpha$.

Test 2. History of stress and suction changes. Model predictions for these values of the transfer coefficient $\alpha$.

On suppose que les succions macrostructurale et microstructurale sont initialement en équilibre. Le changement de la succion macrostructurale (de $90 \mathrm{MPa}$ à $0 \mathrm{MPa}$ ) provoque un phénomène transitoire de transfert d'eau entre les vides de la macrostructure et les pores des particules de roche qui peut être observé sur la figure 25 (évolution de la succion microstructurale). Ce phénomène transitoire est contrôlé par la valeur du paramètre $\alpha$. Trois cas ont été représentés sur la figure 25 . 
Pour une forte valeur de $\alpha$ (Cas C: $\alpha=10^{-5} \mathrm{MPa} \mathrm{s}^{-1}$ ), le transfert local d'eau est rapide et les succions macrostructurale et microstructurale évoluent en adoptant presque les mêmes valeurs.

Sur la figure 26, on a représenté les courbes contrainte vertical-déformation mesurées et calculées. Les courbes calculées correspondent aux trois valeurs de (indiquées sur la figure 25 . On peut observer l'effet de ce paramètre sur la réponse mécanique du matériau. Lors de la première réduction de succion (humidification) à contrainte constante $(0,2 \mathrm{MPa})$, la valeur de $\alpha$ contrôle la vitesse de réduction de la succion microstructurale. La réduction étant lente, la réaction de l'échantillon est retardée (faible gonflement). Lorsque la valeur d' $\alpha$ est suffisamment forte, les changements brusques de la succion sur le pourtour de l'échantillon se traduisent en déformations instantanées. Un phénomène similaire est observé pour de plus fortes contraintes appliquées. Lorsque la valeur de $\alpha$ est faible (Fig. 26a), des différences subsistent entre les succions

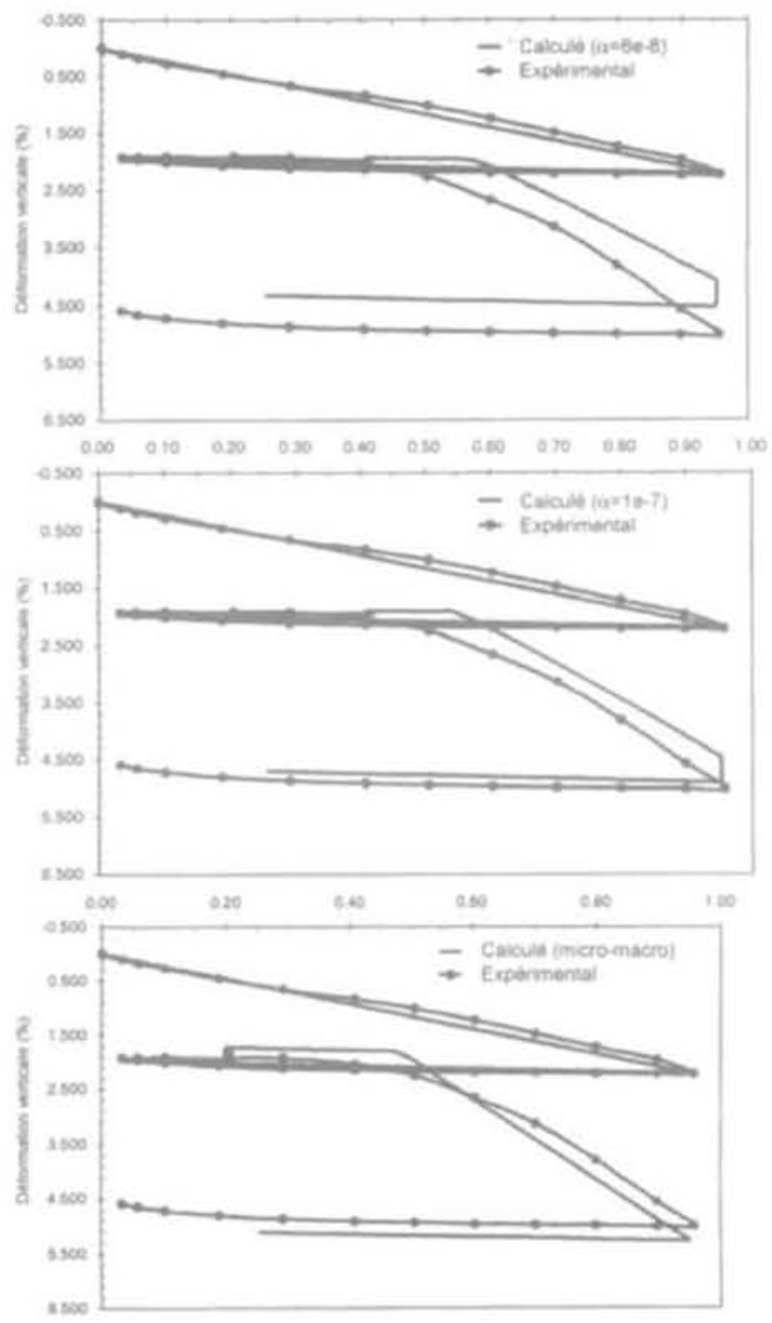

FG. 26 Essai 2. Comparaison entre les résultats expérimentaux et le modèle de calcul.

Test 2. Comparison between experimental results and model calculations. microstructurale et macrostructurale. La première tend lentement à l'équilibre et ce processus provoque des déformations différées à contrainte constante. Ce phénomène s'amortit lorsque $\alpha$ augmente.

Cet exemple manifeste les capacités du modèle à double structure. L'effet « secondaire » de la déformation apparait d'une façon naturelle comme un phénomène de transfert d'eau à l'intérieur des particules de roche. Cet effet temporel n'est pas le seul présent dans les enrochements. En effet, les essais à succion constante (où probablement l'équilibre macro-micro est atteint) montrent une vitesse finie de propagation de fissures et donc de déformations différées. Ce deuxième effet n'a pas été introduit d'une façon explicite dans le modèle développé. La dépendance entre la vitesse de propagation de la rupture et la succion microstructurale semble cohérente avec le mécanisme postulé de propagation subcritique de fissures. Il est raisonnable de penser que l'énergie de l'eau qui sature partialement les fissures actives des particules contrôle leur propagation. La succion micro ainsi définie est une approximation de la succion existant réellement aux extrémités des fissures actives.

\section{6}

\section{Conclusion}

Le mécanisme de propagation subcritique des fractures fournit un cadre adéquat au développement d'un modèle constitutif de comportement des enrochements qui considère l'action de l'eau. Des essais de laboratoire et l'évidence indirecte causée par les observations de terrain démontrent que l'humidité relative est la variable qui contrôle l'effet de l'eau.

Le modèle a été développé en suivant la théorie de la plasticité avec écrouissage. Le modèle a été développé initialement pour expliquer le comportement sous un état isotrope de contraintes puis postérieurement étendu à un état général de contraintes. Dans sa version la plus simple, pour des conditions isotropes de contraintes, il requiert six paramètres rhéologiques qui peuvent être déterminés par des essais de compressibilité œedométrique ou isotrope, préférablement en contrôlant 1'humidité relative.

Un certain parallélisme existe entre le comportement des sols non saturés d'activité modérée et les enrochements. Le modèle élastoplastique, développé par Alonso, Gens et Josa (1990) pour des sols non saturés, présente des similitudes formelles avec le modèle ici présenté. Cependant, des différences fondamentales, montrées dans le tableau I, les séparent.

Un développement additionnel consiste à incorporer les mécanismes de transfert d'eau dans les enrochements. Étant donné que l'état d'humidité des particules de roche a une influence directe sur le comportement global, on a développé un modèle pour considérer l'échange d'humidité à échelle locale. La rupture des particules est en rapport avec la succion ou énergie de l'eau à l'intérieur des particules de roche. La méthode décrite permet d'inclure naturellement les effets observés de déformation secondaires. Le modèle couplé écoulement-déformation est un outil puissant pour simuler la réponse de barrage ou remblai en enrochement soumis à des changements de leur environnement. 
TABLEAUI Comparaison entre le comportement des sols non saturés et celui des enrochements.

Comparison between unsaturated soil and rockfill behaviour.

\section{Enrochement}

Sol non saturé

L'effondrement est lié à la rupture de particules et au rẻarrangement postérieur de la structure granulaire.

La ténacité des particules est une propriêtể fondamentale.

L'effet de la succion est de contròler la vitesse de rupture des particules.

La tẻnacité des particules apparaît dans le modèle à travers le paramètre $(p)$. Si la contrainte movenne $p<p$ il n'y a pas de déformations différées ou de phénomène d'effondrement.

\begin{tabular}{|c|c|}
\hline La succion totale contrōle les effets de l'eau. & La succion matricielle ou capillaire contrôle les effets de l'eau \\
\hline $\begin{array}{l}\text { Les déformations différées (et donc l'effondrement) sont inexis- } \\
\text { tantes pour les états très secs. }\end{array}$ & Il n'y a pas de concepts équivalents dans les sols. \\
\hline $\begin{array}{l}\text { Le seuil de plasticité pour un état très sec est un choix conve- } \\
\text { nable comme paramétre d'écrouissage. }\end{array}$ & $\begin{array}{l}\text { Le seuil de plasticité de l'ẻtat saturé est un choix convenable } \\
\text { comme paramètre d'écrouissage. }\end{array}$ \\
\hline $\begin{array}{l}\text { Les déformations élastoplastiques (instantanées et différées) } \\
\text { sont en rapport linéaire avec les contraintes de confinement } \\
\text { (pour l'intervalle de contraintes habituellement considérés en } \\
\text { pratique). }\end{array}$ & $\begin{array}{l}\text { Les déformations élastoplastiques sont en rapport linéaire avec } \\
\text { le logarithme de la contrainte de confinement. }\end{array}$ \\
\hline $\begin{array}{l}\text { Dans les matériaux compactés, la surface de charge correspond } \\
\text { initialement à un état de contrainte de confinement nulle. }\end{array}$ & $\begin{array}{l}\text { Les matériaux compactés se caractérisent par une surface ini- } \\
\text { tiale de charge. }\end{array}$ \\
\hline
\end{tabular}

L'effondrement est lié au réarrangement des particules du sol.

La ténacité ou la résistance des particules n'affecte pas le comportement

L'effet de la succion est d'introduire une contrainte interne de précompression.

Il n'y a pas de concepts équivalents dans les sols.

tiale de charge.

\section{Bibliographie}

Alonso E.E., Gens A. \& Hight D.W. - « Spe: cial Problem Soils. General Report $x$. Eur. Conf. On Sail Mech. And Found. Engng. Dublin, 1987

Alonso E.E., Batlle F. Gens A. \& Lloret A. "Consolidation analysis of partially saturated siols. Application to earthdam construction $x$. Proceedings of the 6th Int. Conf. on Num. Meth, in Geomech. Innsbruck, 1988, p. 1303-1308.

Alonso E. E. Gens A. \& Josa A. - «A constitutive model for partially saturated soils r. Géotechnique, 40, 1990, p. 405430

Alonso E.E., Batlle F., Gens A., L.loret A. \& Delahaye C. - « Análisis de la construcción y llenado de presas de materiales sueltos v. Simp. Geotecnia de Presas de Materiales Sueltos, SEMS, Zaragoza, 1993, p. 191-204.

Alonso E.E. \& Oldecop L.A. - « Fundamentals of rockfill collapse 1 . 1st Asian Conference on Unsat. Soils, Singapore, 2000.

Atkinson B.K. \& Meredith P.G. - $\alpha$ The theory of subcritical crack growth with applications to minerals and rocks $x$. Fracture Mechanics of Rock. B.K. Atkinson ed. London, Academic Press Inc., 1987, p. 111-166.

Bauman P. - Rockfill dams: Cogswell and San Gabriel Dams. Transactions of the ASCE, 125 (2), 1960, p. 29-57.
Broek D. - Elementary engineering fracture mechanics. Dordrecht, Martinus Nihoff Publishers, 1986.

Cetin H., Laman M. \& Ertunç A. - t Settlement and slaking problems in the world's fourth largest rock-fill dam, the Ataturk Dam in Turkey n. Engineering Geology 56. 2000, p. 225-242.

Clements R.P. - The deformation of rockfill: inter-particle behaviour, bulk properties and behaviour in dams. Ph. D. Thesis. Faculty of Engineering, King's College, London Liniversity, 1981.

Coussy O. - Mechanics of porous contirua Chichester, John Wiley \& Sons Ltdi. 1995.

De Alba E, \& Sesana F. - «The influence of expansive minerals on basalt behaviour p. Proc. Int. Congress Engng Geology, Madrid. 1978, p. 107-116.

Delgado I. Veiga Pinto A. \& Maranha das Neves $E$ - Rock index properties for prediction of rockfill behaviour. Memoria $n^{\circ}$ 581, Laboratório Nacional de Engenharia Civil, Lisbon, 1982.

Freiman S.W. - «Effects of chemical environment s on slow crack growth in glasses and ceramics 1). J. Geophysical Research, 89 (B6), 1984, p. 4072-4076.

Gens A. \& Potts D.M. - "Critical state models in computational geomechanics s. Engineering Computations Journal, 1988, p. $178-197$
Gill J. - Modelo microestructural para medios granulares no saturados. Tesis Doctoral. UPC. Barcelona, 1988

Justo J.L. \& Durand P, - u Settlement-time behaviour of granular embankments in. Int. J. For Numerical and Analytical Methods in Geomechanics, 24, 1999, p. $281-303$

Marachi N.D. Chan C.K. Seed H.B. \& Duncan J.M. - Strength and deformation. characteristics of rockfill materials. Dep. Of Civil Engng. Report n ${ }^{2}$ TE-69-5, University of California, 1969

Marsal R.f. - « Mechanical properties of rockfill 1). Embankment Dam Encineering. Casagrande Volume. Hirschfeld R.C. \& Poulos S.J.. eds. John Wiley \& Sons, 1973

Marsal R.J., Arellano L.R. Guzmásn M.A. \& Adame H, - «Fl infernillo n. Behaviour of dams buitt in Mexico. Mexico, Instituto de Ingenieria. UNAM. 1976.

Michalske T.A. \& Freiman S.W. - " A molecular interpretation of stress corrosion in sillica n. Nature, 295, 1982, p. 511-512.

Naylor D I Maranha das Neves E Mattar D. \& Veiga Pinto A.A. - «Prediction of construction performance of Beliche Dam x. Géotechnique, 36 (2), 1986, p. 221233.

Naylor D.J., Maranha J.R., Maranha das Neves E. \& Veiga Pinto A.A. - «A backanalysis of Beliche Dam x. Géotechnique 48 (2). 1997, p. $221-233$ 
Nobari E.S. \& Duncan J.M. - Effect of reservoir filling on stresses and movements in earth and rockfill dams. Department of Civil Engineering, Report $n^{\circ} \mathrm{TE}$ 72-1. University of California, 1972.

Oldecop L.A. \& Alonso E.E. - «A model for rockfill compressibility n, Géotechnique; 51, 2, 2001, p. 127-139.

Olivella S., Carrera J., Gens A. \& Alonso E.E. $-\ll$ Nonisothermal multiphase flow of brine and gas through saline media n. Transport in Porous Media 15, 1994 , p. 271-293.

Ollvella S., Gens A. \& Josa A. - « Numerical formulation for a simulator (CODE_BRIGHT) for the coupled analysis of saline media m. Engineering Computations, 13, 7, 1996, p. 87-112.

Ohmaki S. - «Stress-strain behaviour of anisotropicaliy, normaliy consolidated cohesive soil $x$. Proceedings 1st Int.
Symp. Num. Methods on Geomechanics, Zurich, 1982, p. 250-269.

Rein J.A., Soriano A. \& Pradera E. - «Estabilidad de los terraplenes del ferrocarril de alta velocidad del NAFA. III $v$, Simposio Nacional sobre Taludes y Laderas Inestables, La Coruña, 1992, p. 509-520. Schnitter N.J. - A history of dams. The useful pyramids. Rotterdam, Balkema, 1994. Sherard J.L_\& Cooke J.B, - "Concrete-face rockfill dam: L. Assessment n, J. of Geotech. Engng. ASCE, 113 (10), 1987, p. 1096-1112.

Soriano A. \& Sánchez. F.J, - "Settlements of railroad high embankments n. Proc. XII European Cont. on Soil Mech. and Geotech. Eng., Netherlands, 1999. Sowers G.F., Williams R.C. \& Wallace T.S. - * Compressibility of broken rock and settlement of rockfills ${ }^{2}$. Proc. 6th ICSMFE, 2, Montreal, 1965, p. 561-565.
Terzaghi K. - " Discussion on salt springs and lower bear river dams . Trans. ASCE, 125 (2), 1960, p. 139-148.

Veiga Pinto A.A. - Previsao do comportamento de enrocamento. PhD Thesis. Lisboa: Laboratorio Nacional de Engenheria Civil, 1983.

Viñas J.M. - « Mecanismo fiscoquímico del fenómeno que origina el colapso de los materiales pétreos $n$. Bol. Lab. Transp. Yy. $\mathrm{Mec}$. del Suelo, 100, 1973, p. 71-79.

Vutukuri V.S. \& Lama R.D. - Handbook on Mechanical Properties of Rocks. Claustahl, Trans Tech Publications, 1978. Wiederhorn S.M. Fuller E.R. \& Thomson R. $-\pi$ Micro-mechanisms of crack growth in creamics and glasses in corrosive environments v. Met. Scl. 14, 1980, p.450-458.

Wiederhorn S.M. Freiman S.W... Fuller E.R. \& Simmons C.J. - \& Effects of water and other dielectrics on crack growth $x$. I. Mater. Sci.. 17, 1982, p. 3460. 